\title{
TITLE:
}

\section{Boundary cooperative control by flexible Timoshenko arms}

\author{
$\operatorname{AUTHOR}(\mathrm{S})$ :
}

Endo, Takahiro; Matsuno, Fumitoshi; Jia, Yingmin

\section{CITATION:}

Endo, Takahiro ...[et al]. Boundary cooperative control by flexible Timoshenko arms. Automatica 2017, 81: 377-389

ISSUE DATE:

2017-07

URL:

http://hdl.handle.net/2433/232625

\section{RIGHT:}

(c) 2017. This manuscript version is made available under the CC-BY-NC-ND 4.0 license

http://creativecommons.org/licenses/by-nc-nd/4.0/; The full-text file will be made open to the public on 01 July 2019 in accordance with publisher's 'Terms and Conditions for Self-Archiving'; この論文は出版社版でありません。引用の際に は出版社版をご確認ご利用ください。; This is not the published version. Please cite only the published version. 


\title{
Boundary Cooperative Control by Flexible Timoshenko Arms
}

\author{
Takahiro Endo ${ }^{*}$, Fumitoshi Matsuno ${ }^{\mathrm{a}}$, and Yingmin $\mathrm{Jia}^{\mathrm{b}}$ \\ ${ }^{*}$ Corresponding author. Phone/Fax +81-75-383-3595 \\ ${ }^{\text {a }}$ Department of Mechanical Engineering and Science, Kyoto University, \{endo, matsuno\}@ me.kyoto-u.ac.jp \\ ${ }^{\mathrm{b}}$ Seventh Research Division, Beihang University, ymjia@buaa.edu.cn
}

\begin{abstract}
This paper discusses a cooperative control problem by two one-link flexible Timoshenko arms. The goal is to control a grasping force to collect an object with the two flexible arms, and to simultaneously suppress the vibrations of the arms. To solve this problem, we propose a boundary controller that is based on a dynamic model represented by a hybrid PDE-ODE model; the exponential stability of the closed-loop system is then proven by the frequency domain method. Finally, several numerical simulations are carried out to investigate the validity of the proposed boundary cooperative controller.
\end{abstract}

Key words: Flexible arms, Cooperative control, Force control, Exponentially stable, Distributed parameter systems.

\section{Introduction}

A flexible arm is a controlled system modeled by partial differential equations (PDEs) to present the dynamics of the elastic links, and ordinary differential equations (ODEs) to present the dynamics of sensors, actuators, and tip loads. The dynamic model of the flexible arm is thus a hybrid PDE-ODE model. The Euler-Bernoulli beam model in particular is widely used to present the dynamics of the elastic links. On the other hand, the Timoshenko beam model is a modified model of the Euler-Bernoulli beam model that includes the effects of shear and rotational inertia. Therefore, the Timoshenko beam model can be used in a wider range of applications than the Euler-Bernoulli beam model (Huang, 1961; Han, Benaroya, \& Wei, 1999). Here, the flexible arm, modeled as a Timoshenko beam, is called the flexible Timoshenko arm.

There have been several relevant studies about the flexible Timoshenko arm based on the hybrid PDE-ODE model (Morgül, 1992; Zhang et al., 1997; Taylor \& Yau, 2003; Macchelli \& Melchiorri, 2004; Grobbelaar-Van Dalsen, 2010; He, Zhang, \& Ge, 2013; Muñoz Rivera, \& Ávila, 2015; Endo, Sasaki, \& Matsuno, 2017; and references therein). However, many of these studies on hybrid PDEODE infinite dimensional settings address only the vibration suppression problem. This is insufficient for use of the flexible arm for more complex tasks. In addition to the vibration control, the force control, that is the control of the contact force at the contact point where the flexible arm touches an object or the environment, is necessary for realization of more complex tasks (Yoshikawa, 2000; Hokayen \& Spong, 2006). Very few studies, Endo, Sasaki, and Matsuno (2017) have investigated the force control problem of the flexible Timoshenko arm.
This paper discusses the cooperative control problem of two one-link flexible Timoshenko arms modeled by hybrid PDE-ODE infinite dimensional model. This problem is a typical applied force control problem. In the cooperative control problem, two one-link flexible Timoshenko arms grasp an object, and the flexible arms control the contactforce at the contact point to achieve the desired grasping force. From the point of view of cooperative control, control of grasping force must consider the following: how to control the internal force applied to the grasped object by the two flexible Timoshenko arms, and how to control the motion of the grasped object. For cooperative control of the flexible arms, it is necessary to suppress vibration in the arms, as well as to control the grasping force. Although there has been little research on the cooperative control of flexible arms, several studies have investigated the infinite dimensional settings (Matsuno \& Hayashi, 2000; Morita et al., 2003; Endo, Matsuno, \& Kawasaki, 2009; Dou \& Wang, 2014). These studies (Matsuno \& Hayashi, 2000; Morita et al., 2003; Endo, Matsuno, \& Kawasaki, 2009), discussed the cooperative control by two one-link flexible arms modeled by the Euler-Bernoulli beam model, and proposed asymptotic/exponential stabilizing controllers. Dou and Wang (2014) had a slightly different focus, proposing a cooperative controller for a system in which two rigid arms grasp a flexible object modeled by the EulerBernoulli beam. To the best of our knowledge, however, there has been no study of cooperative control by the flexible Timoshenko arms based on the hybrid PDE-ODE infinite dimensional model. As mentioned above, the Timoshenko beam model is a modified model of the EulerBernoulli beam model, and its application range is wide. Thus, the cooperative control problem by two one-link flexible Timoshenko arms is a challenging and useful issue. 
In this paper, we propose a simple boundary cooperative controller for the cooperative control of two one-link flexible Timoshenko arms. Many boundary controllers have been proposed for various hybrid PDE-ODE infinite dimensional models other than the flexible Timoshenko arm (e.g., Littman \& Markus, 1988; Slemrod, 1989; d'AndréaNovel et al., 1994; Luo, Guo, \& Morgul, 1999; Matsuno, Ohno, \& Orlov, 2002; Choi, Hong, \& Yang, 2004; Yang, Hong, \& Matsuno, 2005; Krstic \& Smyshlyaev, 2008; Nguyen \& Hong, 2010; He et al., 2011; Endo, Matsuno, \& Kawasaki, 2014; Curtain \& Zwart, 2016). In the boundary controller, the control action is at the boundary of the systems. Consideration of the distributed controller of the hybrid PDE-ODE model (e.g. Hong, 1997; He et al., 2016) reveals that implementation of the boundary controller is generally easy from a practical point of view. We therefore propose a boundary controller to solve the cooperative control problem by two one-link flexible Timoshenko arms modeled by the hybrid PDE-ODE model. The proposed system consists of two flexible Timoshenko arms installed on a slider. The two arms push an object toward each other from opposite sides using the same force; together they produce a positive internal force and the object is grasped by the flexible arms. In addition, the motion of the grasped object is realized by the motion of the slider. We prove that cooperative control is accomplished; that is, we prove the exponential stability of the closed-loop system by the frequency domain method. In addition, we consider the robustness of the proposed boundary controller with respect to the disturbance at the grasped object and the control input. The paper is organized as follows: in the next section, we formulate the control problem and propose a simple boundary cooperative controller. Section 3 presents the semigroup setting of the closed-loop system. Exponential stability and the robustness are given in Section 4. The numerical simulation results, which demonstrate the validity of the proposed boundary controller, are presented in Section 5. Finally, Section 6 contains our conclusions.

\section{System description and boundary controller}

\subsection{A controlled system}

Fig. 1 illustrates a controlled system. The system consists of two one-link flexible arms and a grasped object. One end of flexible arm $i, i=1,2$, is clamped to the rotational motor $i$, and the other end has a concentrated mass $m_{i}$. The mass makes contact with a surface of the grasped object. Two rotational motors are installed on the slider; thus, we used three actuators here. This is because, in the vibration control problem of one-link Timoshenko beam, it is well known that a system with one actuator at the free end is exponentially stable if and only if the two wave speeds are equal (this is a physically impossible condition) (Soufyane \& Wehbe, 2003; Almeida Júnior, Santos, \& Muñoz Rivera, 2013). To obtain exponential stability while avoiding this physically impossible condition, it is desirable that the effects of two actuators, such as force and torque, act on one beam. Here, we set two rotational motors and one slider to act as the effects of force and torque

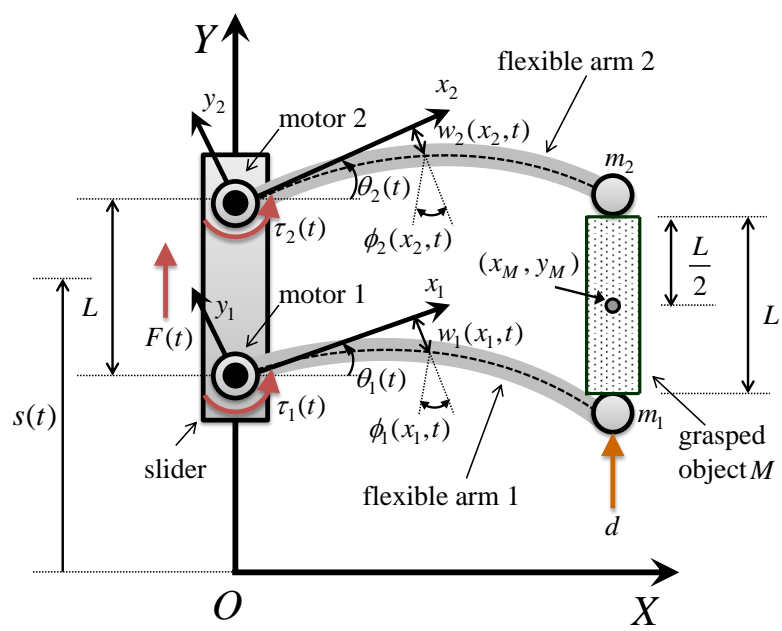

Fig. 1. Grasped object and two flexible Timoshenko arms

at each flexible beam. Using these actuators, the flexible arms rotate and translate in the $X Y$ plane in Fig. 1, and thus it is not affected by the acceleration of gravity. The flexible $\operatorname{arm} i$, with mass per unit length $\rho_{i}$, mass moment of inertial $\mu_{i}$, flexural rigidity $E I_{i}$, cross sectional area $A_{i}$, shear modulus $G_{i}$, shear coefficient $\kappa_{i}$, and length $l$, satisfies the Timoshenko beam hypothesis.

In Fig. 1, $X Y$ is an absolute coordinate system, and $x_{i} y_{i}$ is a local coordinate system, which rotates with the rotor of the motor $i$ and translates with the slider. Let $w_{i}\left(x_{i}, t\right)$ and $\phi_{i}\left(x_{i}, t\right)$ be the transverse displacement and the rotation of the cross section of the flexible arm $i$, respectively, where $x_{i}$ is the spatial point on the local coordinate system $x_{i} y_{i}$, and $t$ is a time. In addition, let $\theta_{i}(t), \tau_{i}(t), J_{i}, s(t), F(t)$, and $M_{s}$ be the angle, torque, and inertial moment of motor $i$, the position of the slider between $O$ and the center of the slider, the force of the slider, and the mass of the slider, respectively. The disturbance $d$ acts at the grasped object, and the disturbance response is discussed in subsection 4.2. For the grasped object, let $M$ and $L$ be the mass and length of the object, respectively, and $\left(x_{M}, y_{M}\right)$ be the position vector of the center of mass in $X Y$. Note that $w_{i}\left(x_{i}, t\right)$, $\phi_{i}\left(x_{i}, t\right), \theta_{i}(t)$, and $s(t)$ are assumed to be small, and the distance between motors 1 and 2 is $L$. Here, note that we make the assumption that the distance between motors 1 and 2 is $L$ to derive the homogeneous boundary conditions. If we do not assume this point, we cannot obtain homogeneous boundary conditions such as (4), and we could not analyze the system. We plan to consider how to eliminate the need for this assumption in future work. In addition, in terms of the contact between the grasped object and flexible Timoshenko arms, we assume the following: the contact is described by a frictional point contact model (Murray, Li, \& Sastry, 1994); there is no slippage and no contact break; the positions of contact points on the $X$-axis are equal to $x_{M}$, and these do not change during movement because both flexible Timoshenko arms are equal in length. Therefore, the flexible arms exert force to the grasped object only in the $Y$ direction, and the grasped object is moved only on the $Y$-axis. 
Since the tip mass makes contact with the surface of the grasped object, we obtain the following geometric constraint:

$\Phi_{i}:=s(t)+l \theta_{i}(t)+w_{i}(l, t)-y_{M}(t)=0, \quad$ for $i=1,2$.

The kinetic energy $E^{k}$ and the potential energy $E^{p}$ of the system are expressed as follows:

$$
\begin{aligned}
& 2 E^{k}= \sum_{i=1}^{2}\left[\rho_{i} \int_{0}^{l}\left\{x_{i} \dot{\theta}_{i}(t)+\dot{s}(t)+\dot{w}_{i}\left(x_{i}, t\right)\right\}^{2} \mathrm{~d} x_{i}+J_{i} \dot{\theta}_{i}^{2}(t)\right. \\
&\left.+\mu_{i} \int_{0}^{l}\left\{\dot{\theta}_{i}(t)+\dot{\phi}_{i}\left(x_{i}, t\right)\right\}^{2} \mathrm{~d} x_{i}+m_{i}\left\{l \dot{\theta}_{i}(t)+\dot{s}(t)+\dot{w}_{i}(l, t)\right\}^{2}\right] \\
&+M_{s} \dot{s}^{2}(t)+M \dot{y}_{M}^{2}(t) \\
& 2 E^{p}=\sum_{i=1}^{2}\left[E I_{i} \int_{0}^{l}\left\{\phi_{i}^{\prime}\left(x_{i}, t\right)\right\}^{2} \mathrm{~d} x_{i}\right. \\
&\left.\quad+K_{i} \int_{0}^{l}\left\{\phi_{i}\left(x_{i}, t\right)-w_{i}^{\prime}\left(x_{i}, t\right)\right\}^{2} \mathrm{~d} x_{i}\right]
\end{aligned}
$$

where $K_{i}:=\kappa_{i} G_{i} A_{i}$, a dot denotes the time derivative, and a prime denotes the partial derivative with respect to the corresponding spatial variable. Further, the virtual work is given by $\delta W=\sum_{i=1}^{2} \tau_{i}(t) \delta \theta_{i}(t)+F(t) \delta s(t)$. Thus, the following equations of motion can be obtained by applying Hamilton's principle and the Lagrange multiplier, and using the same procedures as Endo, Matsuno, and Kawasaki (2009) and Endo, Sasaki, and Matsuno (2017). For $\left(x_{i}, t\right) \in(0, l) \times R^{+}$and $i=1,2$,

$$
\left\{\begin{array}{l}
\rho_{i}\left\{x_{i} \ddot{\theta}_{i}(t)+\ddot{s}(t)+\ddot{w}_{i}\left(x_{i}, t\right)\right\}+K_{i}\left\{\phi_{i}^{\prime}\left(x_{i}, t\right)-w_{i}^{\prime \prime}\left(x_{i}, t\right)\right\}=0, \\
\mu_{i}\left\{\ddot{\theta}_{i}(t)+\ddot{\phi}_{i}\left(x_{i}, t\right)\right\}+K_{i}\left\{\phi_{i}\left(x_{i}, t\right)-w_{i}^{\prime}\left(x_{i}, t\right)\right\}-E I_{i} \phi_{i}^{\prime \prime}\left(x_{i}, t\right)=0, \\
w_{i}(0, t)=\phi_{i}(0, t)=\phi_{i}^{\prime}(l, t)=0, \\
s(t)+l \theta_{i}(t)+w_{i}(l, t)-y_{M}(t)=0, \\
m \ddot{y}_{M}(t)=\sum_{i=1}^{2} K_{i}\left\{\phi_{i}(l, t)-w_{i}^{\prime}(l, t)\right\}, \\
J_{i} \ddot{\theta}_{i}(t)=\tau_{i}(t)+E I_{i} \phi_{i}^{\prime}(0, t), \\
M_{s} \ddot{s}(t)=F(t)-\sum_{i=1}^{2}\left\{\phi_{i}(0, t)-w_{i}^{\prime}(0, t)\right\},
\end{array}\right.
$$

with the algebraic relations

$$
\left\{\begin{array}{l}
m \lambda_{1}(t)=m_{1} f_{2}-\left(M+m_{2}\right) f_{1}, \\
m \lambda_{2}(t)=m_{2} f_{1}-\left(M+m_{1}\right) f_{2},
\end{array}\right.
$$

where $m=M+m_{1}+m_{2}, f_{i}=K_{i}\left\{\phi_{i}(l, t)-w_{i}^{\prime}(l, t)\right\}, i=1,2$, $\lambda_{i}(t)$ is the Lagrange multiplier and is equivalent to the contact force of the flexible arm $i$, which arises in the opposite direction along the normal vector of the constraint surface.

\subsection{Boundary cooperative controller}

The control objective is to construct a boundary controller satisfying: $\lambda_{i}(t) \rightarrow \lambda_{d i}, \quad \dot{w}_{i}\left(x_{i}, t\right) \rightarrow 0, \quad \dot{\phi}_{i}\left(x_{i}, t\right) \rightarrow 0$, $\dot{\theta}_{i}(t) \rightarrow 0, \dot{s}(t) \rightarrow 0$, and $\dot{y}_{M}(t) \rightarrow 0$, where $\lambda_{d i}$ is a constant desired grasping force of the flexible arm $i$. At the equilibrium satisfying the desired conditions $\left(\lambda_{i}(t)=\lambda_{d i}\right.$, $\left.\dot{w}_{i}\left(x_{i}, t\right)=\dot{\phi}_{i}\left(x_{i}, t\right)=\dot{\theta}_{i}(t)=\dot{s}(t)=\dot{y}_{M}(t)=0 \quad\right), \quad w_{i}\left(x_{i}, t\right) \quad$ and $\phi_{i}\left(x_{i}, t\right)$ become a function of $x_{i}$, and $\theta_{i}(t), s(t)$, and $y_{M}(t)$ become constant. We describe them as $w_{d i}\left(x_{i}\right)$, $\phi_{d i}\left(x_{i}\right), \theta_{d i}, s_{d}$, and $y_{M d}$, respectively. By substituting these into (4) and (5), we obtain the following relations: For any given $\lambda_{d 1}, \theta_{d 1}$, and $s_{d}$,

$$
\left\{\begin{array}{l}
w_{d i}\left(x_{i}\right)=\lambda_{d i} x_{i}\left(\frac{1}{K_{i}}+\frac{l x_{i}}{2 E I_{i}}-\frac{x_{i}^{2}}{6 E I_{i}}\right), \\
\phi_{d i}\left(x_{i}\right)=\frac{\lambda_{d i}}{E I_{i}} x_{i}\left(l-\frac{x_{i}}{2}\right), \quad \lambda_{d 2}=-\lambda_{d 1}, \\
\theta_{d 2}=\theta_{d 1}+\frac{1}{l}\left\{w_{d 1}(l)-w_{d 2}(l)\right\}, \\
y_{M d}=s_{d}+l \theta_{d 1}+w_{d 1}(l) .
\end{array}\right.
$$

In particular, in the steady state, if $\theta_{i}=\theta_{d i}$ holds, then we obtain $\lambda_{i}=\lambda_{d i}$.

Based on these results, we construct the controller satisfying

$\left\{\begin{array}{l}w_{i}\left(x_{i}, t\right) \rightarrow w_{d i}\left(x_{i}\right), \quad \dot{w}_{i}\left(x_{i}, t\right) \rightarrow 0, \\ \phi_{i}\left(x_{i}, t\right) \rightarrow \phi_{d i}\left(x_{i}\right), \quad \dot{\phi}_{i}\left(x_{i}, t\right) \rightarrow 0, \quad \text { as } t \rightarrow \infty . \\ \theta_{i}(t) \rightarrow \theta_{d i}, \dot{\theta}_{i}(t) \rightarrow 0, \\ s(t) \rightarrow s_{d}, \quad \dot{s}(t) \rightarrow 0 .\end{array}\right.$

To realize the desired relations (7), we propose the following boundary controller

$$
\begin{gathered}
\tau_{i}(t)=-k_{1 i}\left\{\theta_{i}(t)-\theta_{d i}\right\}-k_{2 i} \dot{\theta}_{i}(t)+k_{3 i} E I_{i} \dot{\phi}_{i}^{\prime}(0, t)-E I_{i} \phi_{d i}^{\prime}(0), \\
F(t)=-k_{4}\left\{s(t)-s^{d}\right\}-k_{5} \dot{s}(t)-k_{6} \sum_{i=1}^{2} K_{i}\left\{\dot{\phi}_{i}(0, t)-\dot{w}_{i}^{\prime}(0, t)\right\},
\end{gathered}
$$

where feedback gains $k_{1 i}, k_{2 i}, k_{3 i}, k_{4}, k_{5}, k_{6}, i=1,2$, are positive constants. The controller (8) is the input to motor $i$, and the controller (9) is the input to the slider. In the controller (8), the first and second terms are PD control of the rotational angle, and the third term is for the vibration suppression. The fourth term is the compensation term of the reaction torque $E I_{i} \phi_{i}^{\prime}(0, t)$. On the other hand, in the controller (9), the first and second terms are PD control of the position control, and the third term is the term of vibration suppression. Here, note that controller (9) has no compensation term of the reaction force because of $\sum_{i=1}^{2} K_{i}\left\{\phi_{d i}(0)-w_{d i}^{\prime}(0)\right\}=0$. With controllers (8) and (9), the effects of the vibration suppression through the force and the torque act at each flexible arm, and thus exponential stability without the physically impossible condition can be expected. In the controller, $\theta_{i}(t)$ and $s(t)$ are measured by the encoder, $\dot{\theta}_{i}(t)$ and $\dot{s}(t)$ are obtained by the numerical difference method, $E I_{i} \phi_{i}^{\prime}(0, t)$ is measured by the strain gauges, and the shear force $K_{i}\left\{\phi_{i}(0, t)-w_{i}^{\prime}(0, t)\right\}$ is obtained by the strain gauges and the difference method (Luo, Kitamura, \& Guo, 1995). So, EI $\dot{\phi}_{i}^{\prime}(0, t)$ and $K_{i}\left\{\dot{\phi}_{i}(0, t)-\dot{w}_{i}^{\prime}(0, t)\right\}$ are obtained by the numerical difference method or by using a speed-reference-type servo amplifier with speed feedback and the high-gain characteristics of the amplifier (Luo, 1993). Therefore, the proposed boundary controller is easily implemented.

\section{Semigroup settings}

Based on (Endo, Matsuno, \& Kawasaki, 2009), let us introduce the following new variables: 


$$
\left\{\begin{array}{l}
y_{1 i}\left(x_{i}, t\right)=w_{i}\left(x_{i}, t\right)+x_{i} \theta_{i}(t)+s(t)-\left\{w_{d i}\left(x_{i}\right)+x_{i} \theta_{d i}+s_{d}\right\}, \\
y_{2 i}\left(x_{i}, t\right)=\phi_{i}\left(x_{i}, t\right)+\theta_{i}(t)-\left\{\phi_{d i}\left(x_{i}\right)+\theta_{d i}\right\}, \\
\eta_{i}(t)=k_{3 i} E I_{i} y_{2 i}^{\prime}(0, t)-J_{i} \dot{y}_{2 i}(0, t)-k_{1 i} k_{3 i} y_{2 i}(0, t), \\
\varsigma(t)=-k_{6} \sum_{i=1}^{2} K_{i}\left\{y_{2 i}(0, t)-y_{1 i}^{\prime}(0, t)\right\}-M_{s} \dot{y}_{11}(0, t) \\
-k_{4} k_{6} y_{11}(0, t) .
\end{array}\right.
$$

Here, $y_{1 i}\left(x_{i}, t\right)$ and $y_{2 i}\left(x_{i}, t\right)$ contribute to the movement of the equilibrium point to its origin, and $\eta_{i}(t)$ and $\varsigma(t)$ are introduced so that the closed-loop system becomes dissipative, as shown in (17). Then, the closed-loop system can be written as follows: For $\left(x_{i}, t\right) \in(0, l) \times R^{+}$and $i=1,2$,

$$
\left\{\begin{array}{l}
\rho_{i} \ddot{y}_{1 i}\left(x_{i}, t\right)+K_{i}\left\{y_{2 i}^{\prime}\left(x_{i}, t\right)-y_{1 i}^{\prime \prime}\left(x_{i}, t\right)\right\}=0, \\
\mu_{i} \ddot{y}_{2 i}\left(x_{i}, t\right)+K_{i}\left\{y_{2 i}\left(x_{i}, t\right)-y_{1 i}^{\prime}\left(x_{i}, t\right)\right\}-E I_{i} y_{2 i}^{\prime \prime}\left(x_{i}, t\right)=0, \\
y_{11}(0, t)=y_{12}(0, t), \quad y_{11}(l, t)=y_{12}(l, t), y_{2 i}^{\prime}(l, t)=0, \\
m \dot{\gamma}(t)=\sum_{i=1}^{2}\left\{y_{2 i}(l, t)-y_{1 i}^{\prime}(l, t)\right\}, \quad \gamma(t)=\dot{y}_{11}(l, t), \\
\dot{\eta}_{i}(t)=k_{1 i} y_{2 i}(0, t)+D_{1 i} \dot{y}_{2 i}(0, t)-E I_{i} y_{2 i}^{\prime}(0, t), \\
\dot{\zeta}(t)=k_{4} y_{11}(0, t)+D_{2} \dot{y}_{11}(0, t)+\sum_{i=1}^{2} K_{i}\left\{y_{2 i}(0, t)-y_{1 i}^{\prime}(0, t)\right\},
\end{array}\right.
$$

where $D_{1 i}=k_{2 i}-k_{1 i} k_{3 i}, D_{2}=k_{5}-k_{4} k_{6}$, and we assumed that $D_{1 i}>0$ and $D_{2}>0$.

Now, we introduce the functional space $H$ as the state space of the closed-loop system: for $z=\left(u_{11}, v_{11}, u_{21}, v_{21}\right.$, $\left.u_{12}, v_{12}, u_{22}, v_{22}, \gamma, \eta_{1}, \eta_{2}, \varsigma\right)^{T}$,

$$
\begin{array}{r}
H=\left\{z \in\left(H^{1}(0, l) \times L^{2}(0, l) \times H^{1}(0, l) \times L^{2}(0, l)\right)^{2} \times C^{4}:\right. \\
\left.u_{11}(0)=u_{12}(0), u_{11}(l)=u_{12}(l)\right\},
\end{array}
$$

where $H^{m}(0, l)$ is the usual Sobolev space of order $m$, $L^{2}(0, l)$ is the usual square integrable functional space, and $C$ is the set of complex numbers. In $H$, we define the inner product: for $z=\left(u_{11}, v_{11}, u_{21}, v_{21}, u_{12}, v_{12}, u_{22}, v_{22}, \gamma, \eta_{1}, \eta_{2}, \varsigma\right)^{T}$ $\in H, \hat{z}=\left(\hat{u}_{11}, \hat{v}_{11}, \hat{u}_{21}, \hat{v}_{21}, \hat{u}_{12}, \hat{v}_{12}, \hat{u}_{22}, \hat{v}_{22}, \hat{\gamma}, \hat{\eta}_{1}, \hat{\eta}_{2}, \hat{\varsigma}\right)^{T} \in H$,

$$
\begin{aligned}
2\langle z, \hat{z}\rangle_{H}=\sum_{i=1}^{2}[ & {\left[\rho_{i} \int_{0}^{l} v_{1 i} \overline{\hat{v}_{1 i}} \mathrm{~d} x_{i}+\mu_{i} \int_{0}^{l} v_{2 i} \overline{\hat{v}_{2 i}} \mathrm{~d} x_{i}+E I_{i} \int_{0}^{l} u_{2 i}^{\prime} \overline{\hat{u}_{2 i}^{\prime}} \mathrm{d} x_{i}\right.} \\
& +K_{i} \int_{0}^{l}\left(u_{2 i}-u_{1 i}^{\prime} \overline{\left(\hat{u}_{2 i}-\hat{u}_{1 i}^{\prime}\right)} \mathrm{d} x_{i}+k_{1 i} u_{2 i}(0) \overline{\hat{u}_{2 i}(0)}\right. \\
& \left.+\frac{1}{J_{i}+k_{3 i} D_{1 i}} \eta_{i} \overline{\hat{\eta}_{i}}+\frac{k_{4}}{2} u_{1 i}(0) \overline{\hat{u}_{1 i}(0)}\right] \\
+ & m \gamma \overline{\hat{\gamma}}+\frac{1}{M_{s}+k_{6} D_{2}} \varsigma \overline{\hat{\zeta}} .
\end{aligned}
$$

It is easy to see that $H$ with the inner product (13) becomes a Hilbert space, because the norm induced by (13) is equivalent to the standard norm in $H$. Further, we define the unbounded linear operator $A: D(A) \subset H \rightarrow H$ as follows:

$$
\begin{aligned}
A z= & {\left[v_{11},-\frac{K_{1}}{\rho_{1}}\left(u_{21}^{\prime}-u_{11}^{\prime \prime}\right), v_{21},-\frac{K_{1}}{\mu_{1}}\left(u_{21}-u_{11}^{\prime}\right)+\frac{E I_{1}}{\mu_{1}} u_{21}^{\prime \prime},\right.} \\
& v_{12},-\frac{K_{2}}{\rho_{2}}\left(u_{22}^{\prime}-u_{12}^{\prime \prime}\right), v_{22},-\frac{K_{2}}{\mu_{2}}\left(u_{22}-u_{12}^{\prime}\right)+\frac{E I_{2}}{\mu_{2}} u_{22}^{\prime \prime}, \\
& \frac{1}{m} \sum_{i=1}^{2} K_{i}\left\{u_{2 i}(l)-u_{1 i}^{\prime}(l)\right\}, k_{11} u_{21}(0)+D_{11} v_{21}(0)-E I_{1} u_{21}^{\prime}(0), \\
& k_{12} u_{22}(0)+D_{12} v_{22}(0)-E I_{2} u_{22}^{\prime}(0), \\
& \left.k_{4} u_{11}(0)+D_{2} v_{11}(0)+\sum_{i=1}^{2} K_{i}\left\{u_{2 i}(0)-u_{1 i}^{\prime}(0)\right\}\right]^{T}
\end{aligned}
$$

with domain

$$
\begin{aligned}
D(A)= & \left\{z \in\left(H^{2}(0, l) \times H^{1}(0, l) \times H^{2}(0, l) \times H^{1}(0, l)\right)^{2} \times C^{4}:\right. \\
& u_{11}(0)=u_{12}(0), u_{2 i}^{\prime}(l)=0, u_{11}(l)=u_{12}(l), \\
& \gamma=v_{11}(l), v_{11}(l)=v_{12}(l), v_{11}(0)=v_{12}(0), \\
& \eta_{i}=k_{3 i} E I_{i} u_{2 i}^{\prime}(0)-J_{i} v_{2 i}(0)-k_{1 i} k_{3 i} u_{2 i}(0), \\
& \left.\varsigma=-k_{6} \sum_{i=1}^{2} K_{i}\left\{u_{2 i}(0)-u_{1 i}^{\prime}(0)\right\}-M_{s} v_{11}(0)-k_{4} k_{6} u_{11}(0)\right\} .
\end{aligned}
$$

Then, the closed-loop system can be written as the following first order evolution equation on $H$ :

$\dot{z}(t)=A z(t), \quad z(0)=z_{0}$,

where $z(t)=\left(y_{11}(\cdot, t), \dot{y}_{11}(\cdot, t), y_{21}(\cdot, t), \dot{y}_{21}(\cdot, t), y_{12}(\cdot, t), \dot{y}_{12}(\cdot, t)\right.$, $\left.y_{22}(\cdot, t), \dot{y}_{22}(\cdot, t), \gamma(t), \eta_{1}(t), \eta_{2}(t), \varsigma(t)\right)^{T}$ is the state, and $z_{0}$ is the initial value.

We obtain the following lemma as the properties of the closed-loop system (16).

Lemma 1: The operator $A$ generates a $C_{0}$-semigroup of contractions. In addition, the operator $A^{-1}$ is compact. Therefore, the spectrum $\sigma(A)$ of the operator $A$ consists only of the isolated eigenvalues.

Proof: First, we show that the operator $A$ is dissipative. From simple calculations using integration by parts and the boundary conditions in (15), we obtain

$$
\begin{aligned}
& 2 \operatorname{Re}\langle A z, z\rangle_{H}=\langle A z, z\rangle_{H}+\langle z, A z\rangle_{H} \\
& =-\sum_{i=1}^{2} \frac{1}{J_{i}+k_{3 i} D_{1 i}}\left[k_{3 i}\left|k_{1 i} u_{2 i}(0)-E I_{i} u_{2 i}^{\prime}(0)\right|^{2}+D_{1 i} J_{i}\left|v_{2 i}(0)\right|^{2}\right] \\
& -\frac{1}{M_{s}+k_{6} D_{2}}\left[\begin{array}{l}
k_{6}\left|k_{4} u_{11}(0)+\sum_{i=1}^{2} K_{i}\left\{u_{2 i}(0)-u_{1 i}^{\prime}(0)\right\}\right|^{2} \\
\left.+M_{s} D_{2}\left|v_{11}(0)\right|^{2}\right] \leq 0,
\end{array}\right.
\end{aligned}
$$

for $z=\left(u_{11}, v_{11}, u_{21}, v_{21}, u_{12}, v_{12}, u_{22}, v_{22}, \gamma, \eta_{1}, \eta_{2}, \varsigma\right)^{T} \in D(A)$. Thus, the operator $A$ is dissipative.

Next, we show $0 \in \rho(A)$, where $\rho(A)$ is the resolvent set of the operator $A$. For any given $\hat{z}=\left(\hat{u}_{11}, \hat{v}_{11}, \hat{u}_{21}\right.$, $\left.\hat{v}_{21}, \hat{u}_{12}, \hat{v}_{12}, \hat{u}_{22}, \hat{v}_{22}, \hat{\gamma}, \hat{\eta}_{1}, \hat{\eta}_{2}, \hat{\zeta}\right)^{T} \in H \quad$, we find a solution $z=\left(u_{11}, v_{11}, u_{21}, v_{21}, u_{12}, v_{12}, u_{22}, v_{22}, \gamma, \eta_{1}, \eta_{2}, \varsigma\right)^{T} \in D(A)$ of the equation $A z=\hat{z}$. Eliminating $v_{1 i}, v_{2 i}, \eta_{i}$, and $\varsigma$ in this equation, we obtain the following: 


$$
\left\{\begin{array}{l}
K_{i}\left\{u_{2 i}^{\prime}\left(x_{i}\right)-u_{1 i}^{\prime \prime}\left(x_{i}\right)\right\}=-\rho_{i} \hat{v}_{1 i}\left(x_{i}\right), \\
K_{i}\left\{u_{2 i}\left(x_{i}\right)-u_{1 i}^{\prime}\left(x_{i}\right)\right\}-E I_{i} u_{2 i}^{\prime \prime}\left(x_{i}\right)=-\mu_{i} \hat{v}_{2 i}\left(x_{i}\right), \\
u_{11}(0)=u_{12}(0), u_{11}(l)=u_{12}(l), u_{2 i}^{\prime}(l)=0, \\
\sum_{i=1}^{2} K_{i}\left\{u_{2 i}(l)-u_{1 i}^{\prime}(l)\right\}=m \hat{\gamma}, \\
k_{1 i} u_{2 i}(0)-E I_{i} u_{2 i}^{\prime}(0)=\hat{\eta}_{i}-D_{1 i} \hat{u}_{2 i}(0), \\
k_{4} u_{11}(0)+\sum_{i=1}^{2} K_{i}\left\{u_{2 i}(0)-u_{1 i}^{\prime}(0)\right\}=\hat{\varsigma}-D_{2} \hat{u}_{11}(0) .
\end{array}\right.
$$

Integrating the first equation of (18) and substituting it into the second equation of (18) leads to

$$
\begin{aligned}
u_{2 i}\left(x_{i}\right)= & -\frac{\rho_{i}}{2 E I_{i}} \int_{0}^{x_{i}}\left(x_{i}-s\right)^{2} \hat{v}_{1 i}(s) \mathrm{d} s+\frac{\mu_{i}}{E I_{i}} \int_{0}^{x_{i}}\left(x_{i}-s\right) \hat{v}_{2 i}(s) \mathrm{d} s \\
& +\frac{c_{1 i}}{2 E I_{i}} x_{i}^{2}+c_{2 i} x_{i}+c_{3 i},
\end{aligned}
$$

where the coefficients $c_{j i}, i=1,2, j=1,2,3$ are constant, determined by the boundary conditions. Further, integrating the first equation of (18) twice, and then using (19) in the obtained equation yields

$$
\begin{aligned}
u_{1 i}\left(x_{i}\right)= & -\frac{\rho_{i}}{6 E I_{i}} \int_{0}^{x_{i}}\left(x_{i}-s\right)^{3} \hat{v}_{1 i}(s) \mathrm{d} s+\frac{\mu_{i}}{2 E I_{i}} \int_{0}^{x_{i}}\left(x_{i}-s\right)^{2} \hat{v}_{2 i}(s) \mathrm{d} s \\
& +\frac{\rho_{i}}{K_{i}} \int_{0}^{x_{i}}\left(x_{i}-s\right) \hat{v}_{1 i}(s) \mathrm{d} s-\frac{c_{1 i}}{K_{i}} x_{i}+\frac{c_{1 i}}{6 E I_{i}} x_{i}^{3} \\
& +\frac{c_{2 i}}{2} x_{i}^{2}+c_{3 i} x_{i}+c_{4 i},
\end{aligned}
$$

where $c_{4 i}$ is constant. Substituting these solutions into the boundary conditions, we obtain the matrix form relation: $M\left[c_{11}, c_{21}, c_{31}, c_{41}, c_{12}, c_{22}, c_{32}, c_{42}\right]^{T}=f$, where $M \in C^{8 \times 8}$ is a matrix, and $f \in C^{8 \times 1}$ is a vector. A simple calculation shows $\operatorname{det} M=-l^{2} k_{4}\left\{k_{11}+k_{12}+\left(E I_{1}+E I_{2}\right) k_{11} k_{12} l /\left(3 E I_{1} E I_{2}\right)\right\} \neq 0$, and thus the coefficients $c_{j i}, i=1,2, j=1, \ldots, 4$, can be uniquely determined. The remaining unknowns, $v_{1 i}, v_{2 i}$, $\eta_{i}$, and $\varsigma$ can be found using $u_{1 i}$ and $u_{2 i}$. Thus, we obtain $0 \in \rho(A)$.

As the operator $A$ is dissipative and $0 \in \rho(A)$, the operator $A$ generates a $C_{0}$-semigroup of contractions from the Lumer-Phillips theorem (Liu \& Zheng, 1999). Finally, we obtain the compactness of the operator $A^{-1}$ as a direct consequence of Sobolev imbedding (Pazy, 1983).

Let $S(t)$ be a $C_{0}$-semigroup of contractions generated by the operator $A$. Lemma 1 means that the closed-loop system (16) has a unique classical solution for $z_{0} \in D(A)$. In addition, the system (16) has a unique mild solution for $z_{0} \in H$, which is the solution of the integral equation $z(t)=A \int^{t} z(s) \mathrm{d} s+z_{0}$ (Engel \& Nagel, 2000). Here, note that the mild solution becomes the classical solution if the operator $A$ generates a differentiable $C_{0}$-semigroup (Pazy, 1983; Luo, Guo, \& Morgul, 1999). To check the differentiability of the semigroup, a spectral analysis of the operator $A$ is required (Pazy, 1983; Liu \& Zheng, 1999). We leave the spectral analysis to show that the mild solution of the operator $A$ becomes the classical solution for future work. If the closed-loop operator $A$ under the boundary controllers (8) and (9) cannot generate a differentiable $C_{0^{-}}$ semigroup, we will propose a boundary controller so that the close-loop operator $A$ generates a differentiable $C_{0^{-}}$ semigroup.

\section{Stability}

\subsection{Exponential stability}

We investigate the exponential stability of the closedloop system (16). Although there are several powerful approaches for investigating exponential stability, such as the Lyapunov functional method and Riesz basis approach, we use the frequency domain method because the calculations are simple. To prove the exponential stability of a $C_{0^{-}}$ semigroup of contractions in a Hilbert space, we need to show the following two facts (Liu \& Zheng, 1999):

(i) $\rho(A) \supset\{i \beta: \beta \in \mathrm{R}\}:=i \mathrm{R}$,

(ii) $\varlimsup_{|\beta| \rightarrow \infty}\left\|(i \beta-A)^{-1}\right\|_{H}<\infty$.

In the following, we show fact (i) in Lemma 2, and (ii) in Lemma 3.

Lemma 2: For the operator $A$, (21) holds.

Proof: We have shown that the spectrum $\sigma(A)$ of the operator $A$ consists only of the isolated eigenvalues in Lemma 1. Thus, to prove (21), we need to show that there are no eigenvalues on the imaginary axis.

Let $s=i \beta$ and $\phi=\left(\phi_{11}, \phi_{21}, \phi_{31}, \phi_{41}, \phi_{12}, \phi_{22}, \phi_{32}, \phi_{42}, \phi_{5}\right.$, $\left.\phi_{61}, \phi_{62}, \phi_{7}\right)^{T} \in D(A)$ be an eigenvalue and the corresponding eigenfunction of the operator $A$, respectively, where $\beta \in \mathrm{R}$. Now, let us consider the eigenvalue problem $A \phi=s \phi$. Noting the fact $0 \in \rho(A)$ in Lemma 1, we can obtain $\operatorname{Re}\langle A \phi, \phi\rangle_{H}=0$, which means the following from (17):

$\left\{\begin{array}{l}k_{4} \phi_{11}(0)+\sum_{i=1}^{2} K_{i}\left\{\phi_{3 i}(0)-\phi_{1 i}^{\prime}(0)\right\}=0, \\ k_{1 i} \phi_{3 i}(0)-E I_{i} \phi_{3 i}^{\prime}(0)=0, \phi_{4 i}(0)=\phi_{21}(0)=\phi_{6 i}=\phi_{7}=0 .\end{array}\right.$

Eliminating the elements of $\phi$ other than $\phi_{1 i}$ and $\phi_{3 i}$ in the equation, $A \phi=s \phi$ leads to:

$$
\left\{\begin{array}{l}
K_{i}\left(\phi_{1 i}^{\prime \prime}-\phi_{3 i}^{\prime}\right)=\rho_{i} s^{2} \phi_{1 i}=0, K_{i}\left(\phi_{1 i}^{\prime}-\phi_{3 i}\right)+E I_{i} \phi_{3 i}^{\prime \prime}=\mu_{i} s^{2} \phi_{3 i}, \\
\phi_{1 i}(0)=\phi_{3 i}(0)=\phi_{3 i}^{\prime}(0)=0, \phi_{3 i}^{\prime}(l)=0, \\
\phi_{11}(l)=\phi_{12}(l), K_{1} \phi_{11}^{\prime}(0)+K_{2} \phi_{12}^{\prime}(0)=0 .
\end{array}\right.
$$

Solving the first, second, and third equations in (24) gives

$$
\left\{\begin{array}{c}
\phi_{1 i}\left(x_{i}\right)=\frac{d}{\sqrt{\omega_{1} \omega_{2}}\left(\omega_{1}-\omega_{2}\right)}\left[\left(b-\omega_{1}\right) \sqrt{\omega_{2}} \sinh \sqrt{\omega_{1}} x_{i}\right. \\
\left.-\left(b-\omega_{2}\right) \sqrt{\omega_{1}} \sinh \sqrt{\omega_{2}} x_{i}\right], \\
\phi_{3 i}\left(x_{i}\right)=\frac{c d}{\omega_{1}-\omega_{2}}\left[\cosh \sqrt{\omega_{1}} x_{i}-\cosh \sqrt{\omega_{2}} x_{i}\right],
\end{array}\right.
$$

where $a=\rho_{i} s^{2} / K_{i}, b=\left(\mu_{i} s^{2}+K_{i}\right) / E I_{i}$, and $c=-K_{i} / E I_{i}$. Further, $\omega_{1}$ and $\omega_{2}$ are roots of $\omega^{2}-(a+b+c) \omega+a b=0$, and we can show that $\omega_{1} \neq \omega_{2}$ using the same procedure as in Han and $\mathrm{Xu}$ (2009). The constant $d$ is determined by 
the remaining boundary conditions. Now, the solutions (25) with the boundary condition $\phi_{3 i}^{\prime}(l)=0$ leads to $\phi_{1 i}=\phi_{3 i}=0$, which means $\phi=0$. This contradicts the fact that $\phi$ is an eigenfunction, and thus the proof is completed.

Lemma 3: For the operator $A$, (22) holds.

Proof: According to the contradiction argument method (Liu and Zheng, 1999), if (22) is false, then there exists a sequence $\beta_{n} \in \mathrm{R}$ with $\beta_{n} \rightarrow \infty$, and a sequence $z_{n} \in D(A)$ with $\left\|z_{n}\right\|_{H}=1$ such that

$\left(i \beta_{n}-A\right) z_{n}:=\phi_{n} \rightarrow 0$ in $H$,

where $z_{n}=\left(u_{11 n}, v_{11 n}, u_{21 n}, v_{21 n}, u_{12 n}, v_{12 n}, u_{22 n}, v_{22 n}, \gamma_{n}, \eta_{1 n}\right.$, $\left.\eta_{2 n}, \varsigma_{n}\right)^{T}, \phi_{n}=\left(\phi_{11 n}, \phi_{21 n}, \phi_{31 n}, \phi_{41 n}, \phi_{12 n}, \phi_{22 n}, \phi_{32 n}, \phi_{42 n}, \phi_{5 n}\right.$, $\left.\phi_{61 n}, \phi_{62 n}, \phi_{7 n}\right)^{T}$. Here, (26) means the following:

$i \beta_{n} u_{1 i n}-v_{1 i n}=\phi_{1 i n}, \quad i \beta_{n} v_{1 i n}+K_{i}\left(u_{2 i n}^{\prime}-u_{1 i n}^{\prime \prime}\right) / \rho_{i}=\phi_{2 i n}$,

$i \beta_{n} u_{2 i n}-v_{2 i n}=\phi_{3 i n}, i \beta_{n} v_{2 i n}+K_{i}\left(u_{2 i n}-u_{1 i n}^{\prime}\right) / \mu_{i}$

$$
-E I_{i} u_{2 i n}^{\prime \prime} / \mu_{i}=\phi_{4 i n} \text {, }
$$

$i \beta_{n} \gamma_{n}-\sum_{i=1}^{2} K_{i}\left\{u_{2 i n}(l)-u_{1 i n}^{\prime}(l)\right\} / m=\phi_{5 n}$,

$i \beta_{n} \eta_{i n}-k_{1 i} u_{2 i n}(0)-D_{1 i} v_{2 i n}(0)+E I_{i} u_{2 i n}^{\prime}(0)=\phi_{6 i n}$,

$i \beta_{n} \varsigma_{n}-k_{4} u_{11 n}(0)-D_{2} v_{11 n}(0)-\sum_{i=1}^{2} K_{i}\left\{u_{2 i n}(0)-u_{1 i n}^{\prime}(0)\right\}=\phi_{7 n}$.

We show the contradictions of $\left\|z_{n}\right\|_{H}=1$, i.e., $\left\|z_{n}\right\|_{H} \rightarrow 0$. For clarity, we divide the proof into four steps.

(1st step) We derive the required estimations for the proof. From the fact that $\phi_{n} \rightarrow 0$ in $H$, we obtain the following:

$$
\left\{\begin{array}{l}
\left\|\phi_{2 i n}\right\|_{L^{2}} \rightarrow 0,\left\|\phi_{4 i n}\right\|_{L^{2}} \rightarrow 0,\left\|\phi_{3 i n}^{\prime}\right\|_{L^{2}} \rightarrow 0, \\
\left\|\phi_{3 i n}-\phi_{1 i n}^{\prime}\right\|_{L^{2}} \rightarrow 0,\left|\phi_{1 i n}(0)\right| \rightarrow 0,\left|\phi_{3 i n}(0)\right| \rightarrow 0, \\
\left|\phi_{6 i n}\right| \rightarrow 0,\left|\phi_{5 n}\right| \rightarrow 0,\left|\phi_{7 n}\right| \rightarrow 0,
\end{array}\right.
$$

where $\|\cdot\|_{L^{2}}^{2}=\int_{0}^{l}|\cdot|^{2} \mathrm{~d} x$. In addition, the estimate

$\left\|\left(u_{1 i}, u_{2 i}\right)\right\|_{2}^{2} \leq \gamma\left\|\left(u_{1 i}, u_{2 i}\right)\right\|_{1}^{2}$, for $\left(u_{1 i}, u_{2 i}\right) \in H^{1}(0, l) \times H^{1}(0, l)$,

and (32) gives

$\left\|\phi_{1 i n}\right\|_{L^{2}} \rightarrow 0,\left\|\phi_{1 i n}^{\prime}\right\|_{L^{2}} \rightarrow 0,\left\|\phi_{3 i n}\right\|_{L^{2}} \rightarrow 0$,

where

$$
\left\{\begin{aligned}
2\left\|\left(u_{1 i}, u_{2 i}\right)\right\|_{1}^{2}=E I_{i}\left\|u_{2 i}^{\prime}\right\|_{L^{2}}^{2}+K_{i}\left\|u_{2 i}-u_{1 i}^{\prime}\right\|_{L^{2}}^{2}+k_{1 i}\left|u_{2 i}(0)\right|^{2} & \\
& +\frac{k_{4}}{2}\left|u_{1 i}(0)\right|^{2} \\
2\left\|\left(u_{1 i}, u_{2 i}\right)\right\|_{2}^{2}=\left\|u_{1 i}\right\|_{L^{2}}^{2}+\left\|u_{1 i}^{\prime}\right\|_{L^{2}}^{2}+\left\|u_{2 i}\right\|_{L^{2}}^{2} & +\left\|u_{2 i}^{\prime}\right\|_{L^{2}}^{2},
\end{aligned}\right.
$$

$\gamma$ is a positive constant, (see Appendix A for proof of estimate (33)). Note that, from the equation $\phi_{1 i n}(l)=\int_{0}^{l} \phi_{1 i n}^{\prime}(s) \mathrm{d} s+\phi_{1 i n}(0)$, the Cauchy-Schwarz inequality, (32), and (34), and the inequality

$|a+b|^{2} \leq(|a|+|b|)^{2} \leq 2\left(|a|^{2}+|b|^{2}\right)$, for $a, b \in \mathrm{C}$, we obtain

$\left|\phi_{\text {lin }}(l)\right| \rightarrow 0$.

On the other hand, we obtain $\operatorname{Re}\left\langle\left(i \beta_{n}-A\right) z_{n}, z_{n}\right\rangle_{H} \rightarrow 0$ from (26). This, with the boundary conditions in (15), means

$$
\left\{\begin{array}{l}
\left|k_{1 i} u_{2 i n}(0)-E I_{i} u_{2 i n}^{\prime}(0)\right| \rightarrow 0, \\
\left|k_{4} u_{11 n}(0)+\sum_{i=1}^{2} K_{i}\left\{u_{2 i n}(0)-u_{1 i n}^{\prime}(0)\right\}\right| \rightarrow 0, \\
\left|v_{2 i n}(0)\right| \rightarrow 0,\left|v_{1 i n}(0)\right| \rightarrow 0,\left|\eta_{\text {in }}\right| \rightarrow 0,\left|\varsigma_{n}\right| \rightarrow 0 .
\end{array}\right.
$$

Finally, from the first equations in (27) and (28), (32) and (38), we have

$\left\{\begin{array}{l}\left|\beta_{n} u_{1 i n}(0)\right| \rightarrow 0,\left|u_{1 i n}(0)\right| \rightarrow 0,\left|\beta_{n} u_{2 i n}(0)\right| \rightarrow 0,\left|u_{2 i n}(0)\right| \rightarrow 0, \\ \left|u_{2 i n}^{\prime}(0)\right| \rightarrow 0,\left|\sum_{i=1}^{2} K_{i} u_{1 i n}^{\prime}(0)\right| \rightarrow 0 .\end{array}\right.$

(2nd step) Combining the two equations in (27) gives

$\beta_{n}^{2} u_{1 i n}-K_{i}\left(u_{2 i n}^{\prime}-u_{1 i n}^{\prime \prime}\right) / \rho_{i}=-i \beta_{n} \phi_{1 i n}-\phi_{2 i n}$.

Multiplying (40) by $\rho_{i}\left(x_{i}-l\right) \overline{u_{1 i n}^{\prime}}$, and integrating it yields

$$
\begin{aligned}
\int_{0}^{l}\left\{\rho_{i} \beta_{n}^{2} u_{1 i n}-K_{i}\left(u_{2 i n}^{\prime}-u_{1 i n}^{\prime \prime}\right)\right\}\left(x_{i}-l\right) \overline{u_{1 i n}^{\prime}} \mathrm{d} x_{i} \\
=-\rho_{i} \int_{0}^{l}\left(i \beta_{n} \phi_{1 i n}+\phi_{2 i n}\right)\left(x_{i}-l\right) \overline{u_{1 i n}^{\prime}} \mathrm{d} x_{i} .
\end{aligned}
$$

Using integration by parts, (39), and the Cauchy-Schwarz inequality, the right-hand side of (41) can be estimated as

$$
\begin{aligned}
& -\rho_{i} \int_{0}^{l}\left(i \beta_{n} \phi_{1 i n}+\phi_{2 i n}\right)\left(x_{i}-l\right) \overline{u_{1 i n}^{\prime}} \mathrm{d} x_{i} \\
& \quad \leq C_{1}\left\{\left\|\phi_{1 i n}\right\|_{L^{2}}+\left\|\phi_{1 i n}^{\prime}\right\|_{L^{2}}\right\}\left\|\beta_{n} u_{1 i n}\right\|_{L^{2}}+C_{2}\left\|\phi_{2 i n}\right\|_{L^{2}}\left\|u_{1 i n}^{\prime}\right\|_{L^{2}},
\end{aligned}
$$

where $C_{1}$ and $C_{2}$ are positive constants. Here note that $\left\|\beta_{n} u_{1 i n}\right\|_{L^{2}}$ is bounded from the first equation in (27), (34), $\left\|z_{n}\right\|_{H}=1$, and the inequality (36). In addition, we obtain the boundedness of $\left\|u_{1 i n}^{\prime}\right\|_{L^{2}}$ from (31) and $\left\|z_{n}\right\|_{H}=1$. Thus, from (41), (32), and (34), we obtain

$$
\int_{0}^{l}\left\{\rho_{i} \beta_{n}^{2} u_{1 i n}-K_{i}\left(u_{2 i n}^{\prime}-u_{1 i n}^{\prime \prime}\right)\right\}\left(x_{i}-l\right) \overline{u_{1 i n}^{\prime}} \mathrm{d} x_{i} \rightarrow 0 .
$$

Here, a simple calculation using the integration by parts and (39) leads to

$$
\begin{aligned}
2 \operatorname{Re} \int_{0}^{l}\left\{\rho_{i} \beta_{n}^{2} u_{1 i n}-K_{i}\left(u_{2 i n}^{\prime}-u_{1 i n}^{\prime \prime}\right)\right\}\left(x_{i}-l\right) \overline{u_{1 i n}^{\prime}} \mathrm{d} x_{i} \\
=-\rho_{i}\left\|\beta_{n} u_{1 i n}\right\|_{L^{2}}^{2}-2 \operatorname{Re} \int_{0}^{l} K_{i}\left(x_{i}-l\right) u_{2 i n}^{\prime} \overline{u_{1 i n}^{\prime}} \mathrm{d} x_{i} \\
\quad+l K_{i}\left|u_{1 i n}^{\prime}(0)\right|^{2}-K_{i}\left\|u_{1 i n}^{\prime}\right\|_{L^{2}}^{2} \rightarrow 0 .
\end{aligned}
$$

Summarizing (44) for $i=1$, and 2, and using (39) gives

$$
\begin{aligned}
-\sum_{i=1}^{2} \rho_{i}\left\|\beta_{n} u_{1 i n}\right\|_{L^{2}}^{2}-2 \sum_{i=1}^{2} \operatorname{Re} \int_{0}^{l} K_{i}\left(x_{i}-l\right) u_{2 i n}^{\prime} \overline{u_{1 i n}^{\prime}} \mathrm{d} x_{i} \\
-\sum_{i=1}^{2} K_{i}\left\|u_{1 i n}^{\prime}\right\|_{L^{2}}^{2} \rightarrow 0 .
\end{aligned}
$$


Similarly, combining the two equations in (28), multiplying the obtained equation by $\mu_{i}\left(x_{i}-l\right) \overline{u_{2 i n}^{\prime}}$, and integrating it, produces a similar calculation

$$
\begin{aligned}
&-\sum_{i=1}^{2}\left(\mu_{i}-\frac{1}{\beta_{n}^{2}}\right)\left\|\beta_{n} u_{2 i n}\right\|_{L^{2}}^{2}+2 \sum_{i=1}^{2} \operatorname{Re} \int_{0}^{l} K_{i}\left(x_{i}-l\right) u_{1 i n}^{\prime} \overline{u_{2 i n}^{\prime}} \\
& \mathrm{d} x_{i} \\
&-\sum_{i=1}^{2} E I_{i}\left\|u_{2 i n}^{\prime}\right\|_{L^{2}}^{2} \rightarrow 0 .
\end{aligned}
$$

Taking the sum of (45) and (46) leads to

$$
\begin{aligned}
\sum_{i=1}^{2}\left\{\rho_{i}\left\|\beta_{n} u_{1 i n}\right\|_{L^{2}}^{2}+K_{i}\left\|u_{1 i n}^{\prime}\right\|_{L^{2}}^{2}+\left(\mu_{i}-\frac{1}{\beta_{n}^{2}}\right)\left\|\beta_{n} u_{2 i n}\right\|_{L^{2}}^{2}\right. \\
\left.+E I_{i}\left\|u_{2 i n}^{\prime}\right\|_{L^{2}}^{2}\right\} \rightarrow 0 .
\end{aligned}
$$

Here, each coefficient is positive, and thus we obtain

$\left\|\beta_{n} u_{1 i n}\right\|_{L^{2}} \rightarrow 0,\left\|u_{1 i n}^{\prime}\right\|_{L^{2}} \rightarrow 0,\left\|\beta_{n} u_{2 i n}\right\|_{L^{2}} \rightarrow 0,\left\|u_{2 i n}^{\prime}\right\|_{L^{2}} \rightarrow 0$.

In addition, from the first equations in (27) and (28), (34), and (48), we obtain

$\left\|v_{\text {lin }}\right\|_{L^{2}} \rightarrow 0,\left\|v_{2 i n}\right\|_{L^{2}} \rightarrow 0$.

(3rd step) We multiply the first equation in (27) by $\rho_{i} \overline{v_{1 i n}}$, and the first equation in (28) by $\mu_{i} \overline{v_{2 i n}}$. Then, the sum of the obtained two equations gives

$$
\rho_{i} \int_{0}^{l} i \beta_{n} u_{1 i n} \overline{v_{1 i n}} \mathrm{~d} x_{i}+\mu_{i} \int_{0}^{l} i \beta_{n} u_{2 i n} \overline{v_{2 i n}} \mathrm{~d} x_{i} \rightarrow 0
$$

using the Cauchy-Schwarz inequality, (34), and (49). Similarly, we multiply the second equation in (27) by $\rho_{i} \overline{u_{1 i n}}$, and the second equation in (28) by $\mu_{i} \overline{u_{2 i n}}$. The sum of the two obtained equations then gives

$$
\begin{gathered}
\rho_{i} \int_{0}^{l} i \beta_{n} v_{1 i n} \overline{u_{1 i n}} \mathrm{~d} x_{i}+K_{i} \int_{0}^{l}\left(u_{2 i n}^{\prime}-u_{1 i n}^{\prime \prime}\right) \overline{u_{1 i n}} \mathrm{~d} x_{i}+\mu_{i} \int_{0}^{l} i \beta_{n} v_{2 i n} \overline{u_{2 i n}} \mathrm{~d} x_{i} \\
+K_{i} \int_{0}^{l}\left(u_{2 i n}-u_{1 i n}^{\prime}\right) \overline{u_{2 i n}} \mathrm{~d} x_{i}-E I_{i} \int_{0}^{l} u_{2 i n}^{\prime \prime} \overline{u_{2 i n}} \mathrm{~d} x_{i} \rightarrow 0
\end{gathered}
$$

using (33), $\left\|z_{n}\right\|_{H}=1$, and (32). Note here that we can easily obtain $\left|u_{j i n}(l)\right| \rightarrow 0$ for $j=1,2$, from the same procedure as derived in (37). Using this estimate, the integration by parts, and (39), (51) can be rewritten as follows

$$
\begin{aligned}
\rho_{i} \int_{0}^{l} i \beta_{n} v_{1 i n} \overline{u_{1 i n}} & \mathrm{~d} x_{i}+K_{i}\left\|u_{2 i n}-u_{1 i n}^{\prime}\right\|_{L^{2}}^{2}+ \\
\mu_{i} \int_{0}^{l} i \beta_{n} v_{2 i n} \overline{u_{2 i n}} & \mathrm{~d} x_{i} \\
& +E I_{i}\left\|u_{2 i n}^{\prime}\right\|_{L^{2}}^{2} \rightarrow 0 .
\end{aligned}
$$

Finally, by taking the sum of (50) and (52), and by taking the real parts of the obtained estimate, we obtain the following

$$
\left\|u_{2 i n}-u_{1 i n}^{\prime}\right\|_{L^{2}} \rightarrow 0, \quad\left\|u_{2 i n}^{\prime}\right\|_{L^{2}} \rightarrow 0 .
$$

(4th step) Multiplying (40) by $\rho_{i} x_{i} \overline{u_{1 i n}^{\prime}}$, and integrating it yields

$$
\int_{0}^{l}\left\{\rho_{i} \beta_{n}^{2} u_{1 i n}-K_{i}\left(u_{2 i n}^{\prime}-u_{1 i n}^{\prime \prime}\right)\right\} x_{i} \overline{u_{1 i n}^{\prime}} \mathrm{d} x_{i} \rightarrow 0
$$

using the same procedure as in the 2nd step and (37). Taking the real parts of (54), the calculation using the integration by parts, and (39) leads to $\rho_{i} l\left|\beta_{n} u_{1 i n}(l)\right|^{2}-2 \operatorname{Re} K_{i} \int_{0}^{l} x_{i} u_{2 i n}^{\prime} \overline{u_{1 i n}^{\prime}} \mathrm{d} x_{i}+K_{i} l\left|u_{1 i n}^{\prime}(l)\right|^{2} \rightarrow 0$.

Noting that $2 \operatorname{Re} K_{i} \int_{0}^{l} x_{i} u_{2 i n}^{\prime} \overline{u_{1 i n}^{\prime}} \mathrm{d} x_{i} \leq l K_{i}\left\|u_{2 i n}^{\prime}\right\|_{L^{2}}^{2}+l K_{i}\left\|u_{1 i n}^{\prime}\right\|_{L^{2}}^{2}$, (48), and (53), we obtain the following from (55):

$\left|\beta_{n} u_{1 i n}(l)\right| \rightarrow 0, \quad\left|u_{\text {lin }}^{\prime}(l)\right| \rightarrow 0$.

From the first equation in (27), (36), (37), and (56), we obtain $\left|v_{1 i n}(l)\right|^{2} \rightarrow 0$, which means

$$
\left|\gamma_{n}\right| \rightarrow 0
$$

from the boundary condition in (15).

Finally, from (49), (53), (39), (38), and (57), we obtain $\left\|z_{n}\right\|_{H} \rightarrow 0$, and this is the contradiction of $\left\|z_{n}\right\|_{H}=1$. Thus, the claim is proved.

Lemmas 2 and 3 summarize the following theorem for the exponential stability of the closed-loop system.

Theorem 1: The closed-loop system (16) is exponentially stable.

Proof: From Lemmas 2 and 3, and the frequency domain method (Liu \& Zheng, 1999), the closed-loop system (16) is exponentially stable.

\subsection{Boundedness to the disturbance at the grasped object}

We now investigate the boundedness of the closed-loop system with respect to the disturbance at the grasped object. This situation is considered, for example, when there is disturbance at the grasped object because the object makes contact with the environment during transportation, and some contact force arises at the object. Here, we assume that the disturbance $d$ acts in the direction of the $Y$-axis (Fig. 1), and that $d$ satisfies the following conditions: $|d| \leq K$ for a positive constant $K$, and $d \in \mathrm{C}^{1}$.

By considering disturbance $d$ at the object, the 6 th equation in (11) becomes

$m \dot{\gamma}(t)=\sum_{i=1}^{2}\left\{y_{2 i}(l, t)-y_{1 i}^{\prime}(l, t)\right\}+d$.

In this case, the closed-loop system (16) can be rewritten as follows:

$\dot{z}(t)=A z(t)+F, \quad z(0)=z_{0}$,

where $F=(0,0,0,0,0,0,0,0, d / m, 0,0,0)^{T}$, and the operator $A$ is the infinitesimal generator of an exponential stable $C_{0^{-}}$ semigroup $T(t)$; that is, $T(t)$ satisfies $\|T(t)\|_{H} \leq M \exp (-\alpha t)$ for some positive constants $M$ and $\alpha$. Here, it is easy to show that $F$ is continuously differentiable on $[0, T]$ for all $t \in[0, T]$ because $d \in \mathrm{C}^{1}$. Thus, the closed-loop system (59) has a unique classical solution for $z_{0} \in D(A)$ and a unique mild solution for $z_{0} \in H$ (Curtain \& Zwart, 1995)

$$
z(t)=T(t) z_{0}+\int_{0}^{t} T(t-s) F(s) \mathrm{d} s .
$$


Now, by taking the norm in (60), we obtain

$$
\|z(t)\|_{H} \leq M \mathrm{e}^{-\alpha t}\left\|z_{0}\right\|_{H}+\frac{K M}{\alpha}\left(1-\mathrm{e}^{-\alpha t}\right) \leq K^{\prime},
$$

where $K^{\prime}$ is a positive constant. Therefore, we found that the solution of the closed-loop system is bounded when the disturbance acts at the grasped object, and the proposed boundary controllers (8) and (9) are robust to such disturbance. Here, note that we could not obtain the relationship between the upper bound $K^{\prime}$ and the gains in the control laws even if we used the other approach described in the next subsection. However, we carry out numerical simulations and investigate the relationships between the gains and the performance of the controller on disturbance response in Section 5. These results give guidance as to how to adjust the gains to improve the performance on disturbance response.

\subsection{Boundedness to the disturbance at the inputs}

On the other hand, it is important to investigate the response of the disturbance at the inputs from a practical point of view. Let $d_{i}, i=1,2$, and $d_{3}$ be the disturbances acting at the input $\tau_{i}(t)$ and $F(t)$, respectively. Further, we assume that $\left|d_{i}\right| \leq K$ for a positive constant $K$, and $d_{i} \in \mathrm{C}^{1}$ for $i=1,2,3$.

In this case, the 5 th and 6th equation in (11) become

$$
\left\{\begin{array}{l}
\dot{\eta}_{i}(t)=k_{1 i} y_{2 i}(0, t)+D_{1 i} \dot{y}_{2 i}(0, t)-E I_{i} y_{2 i}^{\prime}(0, t)-d_{i}, \\
\dot{\zeta}(t)=k_{4} y_{11}(0, t)+D_{2} \dot{y}_{11}(0, t)+\sum_{i=1}^{2} K_{i}\left\{y_{2 i}(0, t)-y_{1 i}^{\prime}(0, t)\right\}-d_{3} .
\end{array}\right.
$$

It is clear that the solution is bounded with respect to the disturbances at the inputs $d_{i}, i=1,2$, and $d_{3}$ using the same procedures described in section 4.2. Now, we investigate the influences of the disturbances to the system.

Let us consider the energy of the system $E(t):=z \|_{H}^{2}$. It is easy to see that the time derivative of $E(t)$ becomes the following from (17):

$$
\begin{aligned}
\dot{E}(t)+ & \sum_{i=1}^{2} \frac{1}{J_{i}+k_{3 i} D_{1 i}}\left[k_{3 i}\left|k_{1 i} u_{2 i}(0)-E I_{i} u_{2 i}^{\prime}(0)\right|^{2}+D_{1 i} J_{i}\left|v_{2 i}(0)\right|^{2}\right] \\
& +\frac{1}{M_{s}+k_{6} D_{2}}\left[k_{6}\left|k_{4} u_{11}(0)+\sum_{i=1}^{2} K_{i}\left\{u_{2 i}(0)-u_{1 i}^{\prime}(0)\right\}\right|^{2}\right. \\
& \left.=-\sum_{i=1}^{2} \frac{1}{J_{i}+k_{3 i} D_{1 i}} \eta_{i} d_{i}-\frac{1}{M_{s}+k_{6} D_{2}} \varsigma d_{3}\left|v_{11}(0)\right|^{2}\right]
\end{aligned}
$$

By integrating both sides with respect to $t$, the right-hand side is estimated as follows:

$$
\begin{aligned}
& -\sum_{i=1}^{2} \frac{1}{J_{i}+k_{3 i} D_{1 i}} \int_{0}^{t} \eta_{i} d_{i} \mathrm{~d} t-\frac{1}{M_{s}+k_{6} D_{2}} \int_{0}^{t} \varsigma d_{3} \mathrm{~d} t \\
& \leq \sum_{i=1}^{2} \frac{1}{J_{i}+k_{3 i} D_{1 i}} \int_{0}^{t}\left[\frac{1}{2 \delta_{i}}\left|\eta_{i}\right|^{2}+\frac{\delta_{i}}{2}\left|d_{i}\right|^{2}\right] \mathrm{d} t \\
& \quad+\frac{1}{M_{s}+k_{6} D_{2}} \int_{0}^{t}\left[\frac{1}{2 \delta_{3}}|\varsigma|^{2}+\frac{\delta_{3}}{2}\left|d_{3}\right|^{2}\right] \mathrm{d} t \\
& \leq \sum_{i=1}^{2} \frac{1}{J_{i}+k_{3 i} D_{1 i}} \int_{0}^{t} \frac{1}{\delta_{i}}\left[k_{3 i}^{2}\left|k_{1 i} u_{2 i}(0)-E I_{i} u_{2 i}^{\prime}(0)\right|^{2}\right. \\
& \quad+\frac{1}{M_{s}+k_{6} D_{2}} \int_{0}^{t} \frac{1}{\delta_{3}}\left[k_{6}^{2} \mid k_{4} u_{11}(0)\right. \\
& \left.\quad+\left.\sum_{i=1}^{2} K_{i}\left\{u_{2 i}(0)-u_{1 i}^{\prime}(0)\right\}\right|^{2}+\left.M_{2 i}^{2}(0)\right|^{2}\right] \mathrm{d} t \\
& \quad+\sum_{i=1}^{2} \frac{1}{J_{i}+k_{3 i} D_{1 i}} \frac{\delta_{i}}{2} \int_{0}^{t}\left|d_{i}\right|^{2} \mathrm{~d} t+\frac{1}{M_{s}+k_{6} D_{2}} \frac{\delta_{3}}{2} \int_{0}^{t}\left|d_{3}\right|^{2} \mathrm{~d} t .
\end{aligned}
$$

Here, we used the inequality $2|a||b| \leq \delta|a|^{2}+|b|^{2} / \delta$ for $a, b \in \mathrm{C}, \delta \in \mathrm{R}^{+}$, to obtain the first inequality. Combining (63) and (64), and setting $\delta_{i}>\max \left\{k_{3 i}, J_{i} / D_{1 i}\right\}$ and $\delta_{3}>\max \left\{k_{6}, M_{s} / D_{2}\right\}$ leads to the following estimation:

$$
\begin{aligned}
E(t) \leq E(0)+\sum_{i=1}^{2} & \frac{1}{J_{i}+k_{3 i} D_{1 i}} \frac{\delta_{i}}{2} \int_{0}^{t}\left|d_{i}\right|^{2} \mathrm{~d} t \\
& +\frac{1}{M_{s}+k_{6} D_{2}} \frac{\delta_{3}}{2} \int_{0}^{t}\left|d_{3}\right|^{2} \mathrm{~d} t,
\end{aligned}
$$

where $E(0)=\left\|z_{0}\right\|_{H}^{2}$. From this estimation, we obtain the following relationships between the disturbances and the gains in the control laws: The effect of the disturbance $d_{i}, i$ $=1,2$, on the $E(t)=\|z\|_{H}^{2}$ can be made small by setting a small $k_{3 i}$ or large $D_{1 i}$ (i.e., a large $k_{2 i}$ and small $k_{1 i}$ ). In addition, the effect of disturbance $d_{3}$ on the $E(t)=\|z\|_{H}^{2}$ can be made small by setting a small $k_{6}$ or large $D_{2}$ (i.e., a large $k_{5}$ and small $\left.k_{4}\right)$. In either case, if we set the gains as stated, the terms about the damping become dominant in the controller, and thus the response of the system becomes slow.

\section{Numerical simulations}

To evaluate the proposed controller, we carried out two numerical simulations. The first is the step responses of the desired grasping force, and the other is the disturbance response. In each case, the numerical simulation is conducted by the finite difference method, and we set $\Delta x_{i}=0.01$ and $\Delta t=0.004$ for the mesh of the spatial variable $x_{i}$ and the time variable $t$, respectively. In addition, to avoid numerical errors, we used the following small parameters: $\rho_{1}=0.016, \rho_{2}=0.018, \mu_{1}=0.025, \mu_{2}=0.028$, $E I_{1}=E I_{2}=K_{1}=K_{2}=l=1, M=0.05, m_{1}=m_{2}=0.025$, $M_{s}=1.5$, and $J_{1}=J_{2}=1$.

\subsection{Step responses}

As the first simulation, we investigated the step responses of the desired grasping force $\lambda_{d 1}=-0.5, \theta_{d 1}=0.5$, and $s_{d}=0.6$. Note here that $\lambda_{d 1}=-0.5$ means that flexible arm 1 pushed the object with a force of $0.5 \mathrm{~N}$. In addition, we compared the proposed controller with the PD controller: 


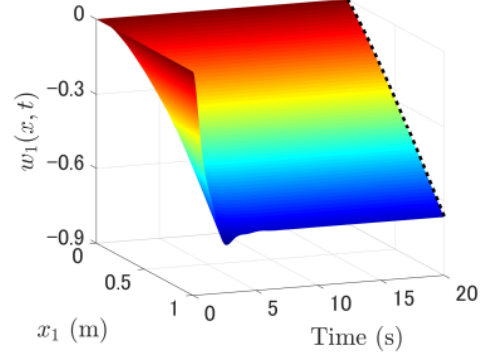

(a) the transverse displacement $w_{1}\left(x_{1}, t\right)$

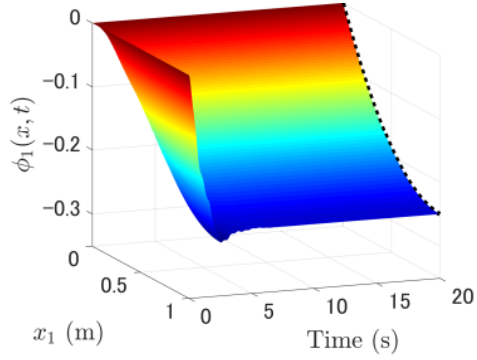

(b) the rotation of the cross section $\phi_{1}\left(x_{1}, t\right)$

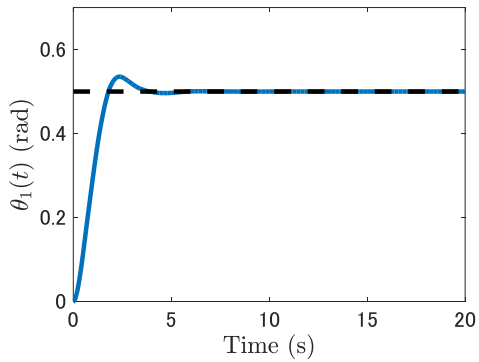

(c) the angle of the motor $\theta_{1}(t)$

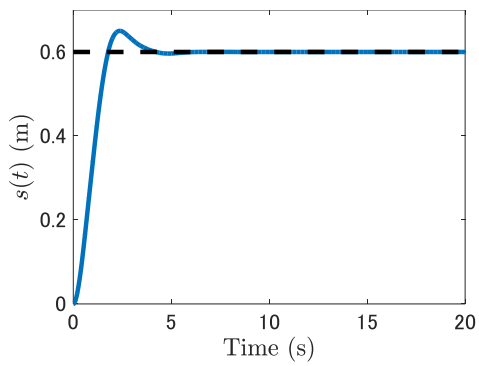

(d) the position of the slider $s(t)$

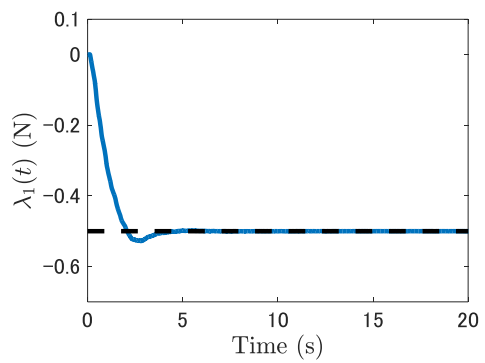

(e) the grasping force $\lambda_{1}(t)$

Fig. 2. Step response of the proposed controller

$$
\tau_{i}(t)=-k_{1 i}\left\{\theta_{i}(t)-\theta_{d i}\right\}-k_{2 i} \dot{\theta}_{i}(t)-E I_{i} \phi_{d i}^{\prime}(0, t),
$$$$
F(t)=-k_{4}\left\{s(t)-s^{d}\right\}-k_{5} \dot{s}(t),
$$

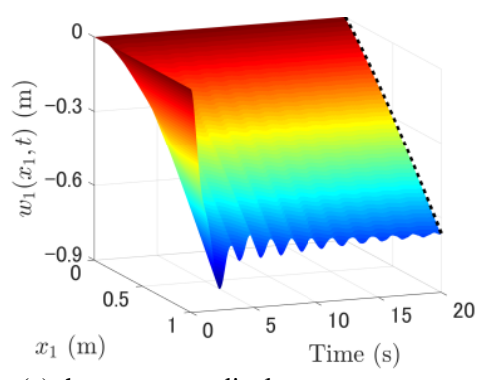

(a) the transverse displacement $w_{1}\left(x_{1}, t\right)$

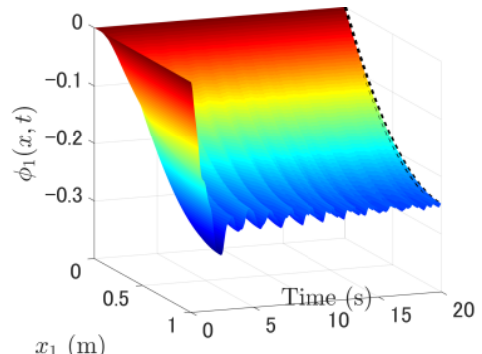

(b) the rotation of the cross section $\phi_{1}\left(x_{1}, t\right)$

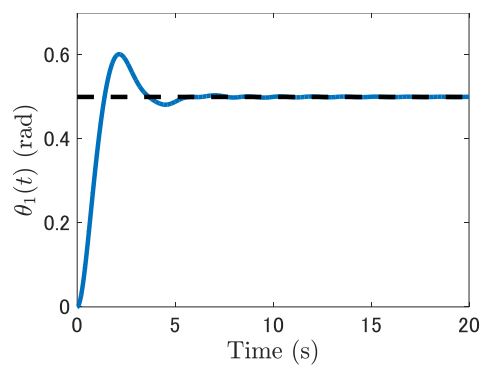

(c) the angle of the motor $\theta_{1}(t)$

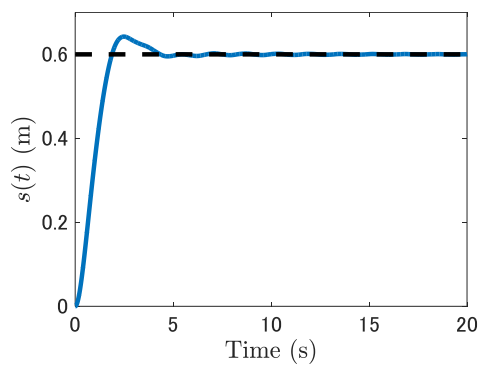

(d) the position of the slider $s(t)$

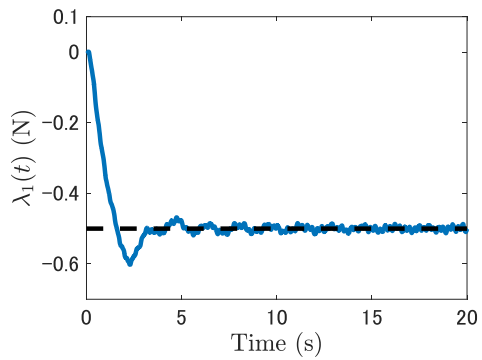

(e) the grasping force $\lambda_{1}(t)$

Fig. 3. Step response of the PD controller

which cannot exponentially stabilize the system because of the result of the compact perturbation (Gibson, 1980; Rao, 1994). As the PD controller, we set the feedback gains as follows: $k_{11}=2, k_{21}=1.7, k_{12}=1.05 k_{11}, k_{22}=1.05 k_{21}$, 


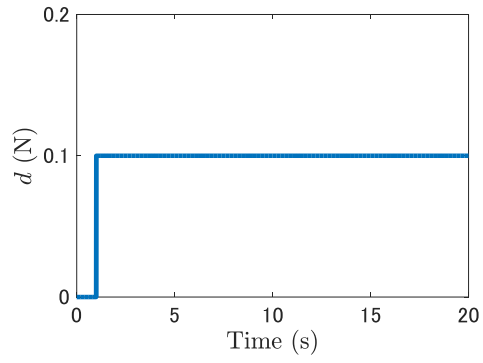

(a) disturbance

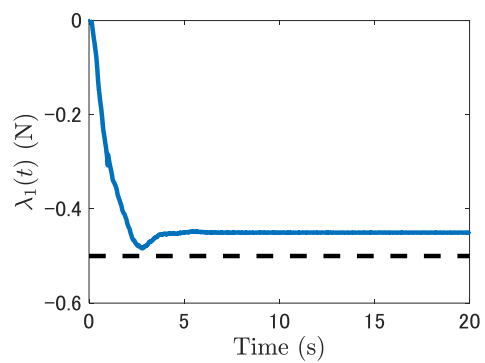

(b) the grasping force $\lambda_{1}(t)$ with the disturbance. The response of

$\lambda_{1}(t)$ without the disturbance is Fig. 2(e).

Fig. 4. Disturbance response of the proposed controller

$k_{4}=4$, and $k_{5}=3.3$. On the other hand, we set the following feedback gains for the proposed controller: $k_{11}=2$, $k_{21}=1.7, k_{12}=1.05 k_{11}, k_{22}=1.05 k_{21}, k_{4}=4, k_{5}=3.3$, $k_{31}=0.8, k_{32}=1.05 k_{31}$, and $k_{6}=0.8$. Figs. 2 and 3 show the step response of the proposed controller and the PD controller, respectively. In particular, as an example, we show the responses of $w_{1}\left(x_{1}, t\right), \phi_{1}\left(x_{1}, t\right), \theta_{1}(t), s(t)$, and $\lambda_{1}(t)$ in each figure. In the figures, the dotted line shows the desired value of the corresponding value. From the simulation results, we found that the $w_{i}\left(x_{i}, t\right)$, $\phi_{i}\left(x_{i}, t\right), \theta_{i}(t), s(t)$, and $\lambda_{i}(t)$ converged to the desired value. Here, note that, not counting the sign (plus or minus) of the value, the responses of $w_{2}\left(x_{2}, t\right), \phi_{2}\left(x_{2}, t\right)$, $\theta_{2}(t)$, and $\lambda_{2}(t)$ were almost the same as the responses of $w_{1}\left(x_{1}, t\right), \phi_{1}\left(x_{1}, t\right), \theta_{1}(t)$, and $\lambda_{1}(t)$, respectively. On the other hand, the responses of the PD controller left some vibrations. Thus, we consider the proposed controller worked well in the step response, and had a higher performance than the PD controller, which could not exponentially stabilize the system.

\subsection{Disturbance responses}

In the next simulation, we confirmed the boundedness of the closed-loop system under the disturbance at the grasped object described in section 4.2. The same feedback gains and physical parameters as in the case of the step responses were used in this simulation. As the disturbance, the settings were that $d=0.1$ acted $1 \mathrm{~s}$ after the start of the simulation:

$d= \begin{cases}0 & (0 \leq t<1) \\ 0.1 & (t>1) .\end{cases}$

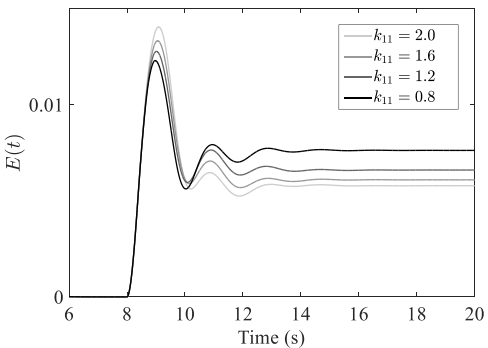

(a) Energies in case (i)

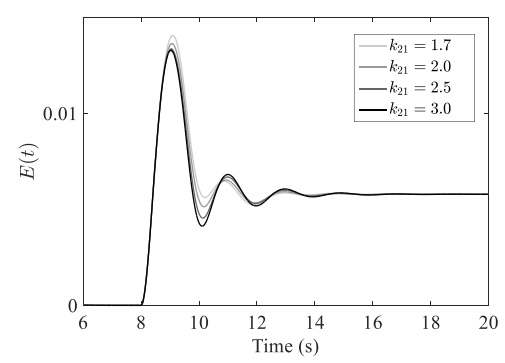

(b) Energies in case (ii)

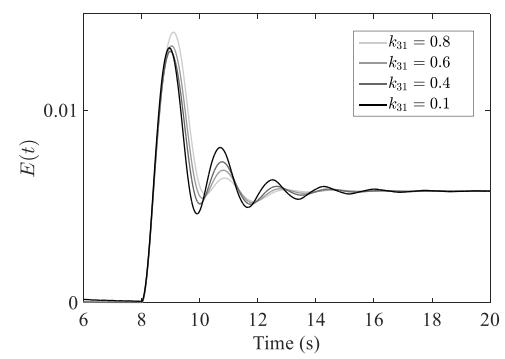

(c) Energies in case (iii)

Fig. 5. Influences of gains in the disturbance response

As an example, we show the response of $\lambda_{1}(t)$ in Fig. 4, where we considered the disturbance response of the proposed controller of $\lambda_{d 1}=-0.5, \theta_{d 1}=0.5$, and $s_{d}=0.6$. In Fig. 4, (a) shows the response of $d$, and (b) shows the response of $\lambda_{1}(t)$ with the disturbance. Note that Fig. 2 (e) is the response of $\lambda_{1}(t)$ without the disturbance. In Fig. 4 (b), the dotted line shows the desired grasping force $\lambda_{d 1}$. From Fig. 2 (e), the response of $\lambda_{1}(t)$ without the disturbance had no steady error; on the other hand, from Fig. 4 (b), it may be seen that the response of $\lambda_{1}(t)$ with the disturbance had a steady error, but did not diverge, and we could confirm the boundedness of $\lambda_{1}(t)$. Of course, we confirmed the boundedness of the other states of the system, and found that the proposed controller was robust with respect to the disturbance at the grasped object.

Next, we investigated the relationship between the feedback gains and performance on disturbance response. The solution of the closed-loop system is estimated as (61) when the disturbance acts at the grasped object. The first term of the right-hand side in (61) corresponds to the initial response, and the second term to the disturbance response. To emphasize the disturbance response, we carried out simulations using the same physical parameters and the following disturbance: 
$d= \begin{cases}0 & (0 \leq t<8) \\ 0.1 & (t>8)\end{cases}$

As the feedback gains of the proposed controller, we set $k_{1 i}=2.0, k_{2 i}=1.7$, and $k_{3 i}=0.8$, for $i=1,2, k_{4}=4.0$, $k_{5}=3.3$, and $k_{6}=0.8$, and we show the simulation results for the following three cases: case (i) $k_{11}$ was changed as follows: $k_{11}=2.0,1.6,1.2,0.8$, while other gains were not changed; case (ii) $k_{21}$ was changed as follows: $k_{21}=1.7,2.0,2.5,3.0$ while other gains were held steady; and case (iii) $k_{31}$ was changed as follows: $k_{31}=0.8,0.6,0.4,0.1$, while other gains were not changed. Here the changing ranges of the gains were set so that $D_{1 i}>0$ and $D_{2}>0$ were satisfied.

Figs. 5(a), (b), and (c) show the response of the energy $E(t)=\|z\|_{H}^{2}$ in cases (i), (ii), and (iii), respectively. From Fig. 5(a), it may be seen that the steady error caused by the disturbance was reduced when $k_{11}$ was large, but the upper bound of the response increased. Fig. 5(b) shows that the upper bound was small when $k_{21}$ was large. However, note that we could not set $k_{21}$ to be too large, because $\dot{\theta}_{i}(t)$ was usually obtained by the numerical difference. On the other hand, the steady error did not change as $k_{21}$ was changed. Fig. 5(c) shows that the upper bound became small when $k_{31}$ was small, but became large when $k_{31}$ was too small, because the small $k_{31}$ led to large vibration in the response. On the other hand, the steady error did not change as $k_{31}$ was changed. Here, note that we obtained the same results for the cases in which the other gains were changed; that is, $k_{12}$ and $k_{4}$ had the same performance as $k_{11} ; k_{22}$ and $k_{5}$ had the same performance as $k_{21} ; k_{32}$ and $k_{6}$ had the same performance as $k_{31}$. These results give the following guidance as to how to adjust the gains to the disturbance at the grasped object: to obtain a small steady error, large $k_{1 i}$ and $k_{4}$ are required while $D_{1 i}>0$ and $D_{2}>0$ are maintained. To obtain a small upper bound, we need a large $k_{2 i}$ and $k_{5}$ or small $k_{3 i}$ and $k_{6}$ while maintaining $D_{1 i}>0$ and $D_{2}>0$, and while noting the vibration of the response.

\section{Conclusions}

In this paper, we considered a cooperative control problem by two one-link flexible Timoshenko arms. To solve the cooperative control problem, we proposed a boundary cooperative controller based on the hybrid PDE-ODE model. The proposed controller has a simple structure, and is thus easy to implement. Further, to obtain exponential stability without the physically impossible condition, we set the design so that the control actuator consisted of the motor and slider, and the effects of force and torque acted at each flexible beam. The exponential stability of the closed-loop system was proven by the frequency domain method. In addition, we investigated the robustness of the proposed controller with respect to the disturbance at the grasped object and the control input. Finally, in numerical simulations, we confirmed that the proposed controller works well for the step and disturbance responses, and the controller has good performance.
In this paper, we considered a fixed contact model between the flexible arm and grasped object. To make the controller more practical, other contact modes, including rolling contact, are desirable, and we plan to propose such a controller for the cooperative control problem using flexible Timoshenko arms in future research.

\section{Acknowledgements}

This paper was supported in part by JSPS KAKENHI Grand Number 23700143 and by the Major International Joint Research Project of NSFC under grant no 61520106010 .

\section{References}

Almeida Júnior, D.S., Santos, M.L., \& Muñoz Rivera, J.E. (2013) Stability to weakly dissipative Timoshenko systems, Mathematical Methods in the Applied Sciences, 36(14), 1965-1976.

d'Andréa-Novel, B., Boustany, F., Conrad, F., \& Rao, B.P. (1994). Feedback stabilization of a hybrid PDE-ODE system: Application to an overhead crane, Math. Control Signals Syst., 7(1), 122.

Choi, J.Y., Hong, K.-S., \& Yang, K.-J. (2004). Exponential stabilization of an axially moving tensioned strip by passive damping and boundary control. J. Vib. Control, 10(5), 661-682.

Curtain, R.F., \& Zwart, H.J. (1995). An introduction to infinitedimensional linear systems theory. Springer-Verlag New York.

Curtain, R., \& Zwart, H. (2016). Stabilization of collocated systems by nonlinear boundary control. Systems \& Control Letters, 96, 11-14.

Dou, H., \& Wang, S. (2014). A boundary control for motion synchronization of a two-manipulator system with a flexible beam, Automatica, 50(12), 3088-3099.

Endo, T., Matsuno, F., \& Kawasaki, H. (2009). Simple boundary cooperative control of two one-link flexible arms for grasping, IEEE Trans. Autom. Control., 54(10), 2470-2476.

Endo, T., Matsuno, F., \& Kawasaki, H. (2014). Force Control and Exponential Stabilization of One-Link Flexible Arm, International Journal of Control, 87(9), 1794-1807.

Endo, T., Sasaki, M., \& Matsuno, F. (2017). Contact-Force Control of a Flexible Timoshenko Arm, IEEE Trans. Autom. Control., 62(2), 1004-1009.

Engel, K.-J., \& Nagel, R. (2000). One-Parameter Semigroups for Linear Evolution Equations. Springer-Verlag New York.

Gibson, J.S. (1980). A note on stabilization of infinite dimensional linear oscillators by compact linear feedback, SIAM J. of Control and Optimization, 18(3), 311-316.

Grobbelaar-Van Dalsen, M. (2010). Uniform stability for the Timoshenko beam with tip load, J. Math. Anal. Appl., 361(2), 392-400.

Han, S.M., Benaroya, H., \& Wei, T. (1999). Dynamics of transversely vibrating beams using four engineering theories. $J$. Sound Vib., 225(5), 935-988.

Han, Z.J., \& Xu, G.Q. (2009). Stabilization and Riesz Basis Property of Two Serially Connected Timoshenko Beams System. Z. Angew. Math. Mech., 89(12), 962-980.

Hong, K.-S. (1997). Asymptotic behavior analysis of a coupled time-varying system: Application to adaptive systems. IEEE Trans. Autom. Control, 42(12), 1693-1697.

He, W., Ge, S.S., How, B.V.E., Choo, Y.S., \& Hong, K.-S (2011). Robust adaptive boundary control of a flexible marine riser with vessel dynamics. Automatica, 47(4), 722-732.

He, W., Zhang, S., \& Ge, S.S. (2013). Boundary output-feedback stabilization of a Timoshenko beam using disturbance observer, IEEE Trans. Ind. Electron., 60(11), 5186-5194.

He, W., Yang, C., Meng, T., \& Sun, C. (2016). Distributed control of a class of flexible mechanical systems with global constraint. International Journal of Control, 89(1), 128-139. 
Hokayem, P.F., \& Spong, M.W. (2006). Bilateral teleoperation: An historical survey, Automatica, 42(12), 2035-2057.

Huang, T.C. (1961). The effect of rotary inertial and of shear deformation on the frequency and normal mode equations of uniform beams with simple end conditions. J. Appl. Mech., 28(4), $579-584$.

Krstic, M., \& Smyshlyaev, A. (2008). Boundary Control of PDEs. SIAM.

Littman, W., \& Markus, L. (1988). Stabilization of a hybrid system of elasticity by feedback boundary damping. Annu. Mat. Pura Appl., 152(1), 281-330.

Liu, Z., \& Zheng, S. (1999). Semigroups associated with dissipative systems. Chapman and Hall/CRC.

Luo, Z.H. (1993). Direct strain feedback control of flexible arms: New theoretical and experimental results, IEEE Trans. Autom. Control., 38(11), 1610-1622.

Luo, Z.-H., Guo, B.-Z., \& Morgul, O. (1999). Stability and Stabilization of Infinite Dimensional Systems with Applications. Springer-Verlag London Limited.

Luo, Z.H., Kitamura, N., \& Guo, B.Z. (1995). Shear force feedback control of flexible robot arms, IEEE Trans. Robot. Automat., 11(5), 760-765.

Matsuno, F., \& Hayashi, A. (2000). PDS cooperative control of two one-link flexible arms, Proc. of the 2000 IEEE Int. Conf. Robotics \& Automation, San Francisco, CA (pp.1490-1495).

Matsuno, F., Ohno, T., \& Orlov, Y.V. (2002). Proportional derivative and strain (PDS) boundary feedback control of a flexible space structure with a closed-loop chain mechanism. Automatica, 38(7), 1201-1211.

Morgül, Ö. (1992). Dynamic boundary control of the Timoshenko beam. Automatica, 28(6), 1255-1260.

Morita, Y., Matsuno, F., Ikeda, M., Ukai, H., \& Kando, H. (2003). PDS cooperative control of two one-link flexible arms considering bending and torsional deformation, Proc. of the 29th Ann. Conf. of the IEEE Industrial Electronics Society, (pp.466-471).

Muñoz Rivera, J.E., \& Ávila, A.I. (2015). Rates of decay to non homogeneous Timoshenko model with tip body, J. Differential Equations, 258(10), 3468-3490.

Murray, R.M., Li, Z., \& Sastry, S.S. (1994). A Mathematical Introduction to Robotic Manipulation. CRC Press LLC.

Nguyen, Q.C., \& Hong, K.-S. (2010). Asymptotic stabilization of a nonlinear axially moving string by adaptive boundary control. J. Sound Vib., 329(22), 4588-4603.

Pazy, A. (1983). Semigroups of linear operators and applications to partial differential equations. Springer-Verlag New York.

Rao, B. (1994). Recent progress in non-uniform and uniform stabilization of the SCOLE model with boundary feedbacks. In J. P. Zolésio (ed.), Bounday control and variation (pp.357-365), Marcel Dekker New York.

Slemrod, M., (1989). Feedback stabilization of a linear control system in Hilbert space with an a priori bounded control, Math Control Signals Syst., 2(3), 265-285.

Soufyane, A., \& Wehbe, A. (2003). Uniform stabilization for the Timoshenko beam by a locally distributed damping, Electron. J. Differential Equations, 29, 1-14.

Taylor, S.W., \& Yau, S.C.B. (2003). Boundary control of a rotating Timoshenko beam, J. ANZIAM, 44, E143-E184.

Yang, K.-J., Hong, K.-S., \& Matsuno, F. (2005). Boundary control of a translating tensioned beam with varying speed. IEEE/ASME Trans. Mechatronics, 10(5), 594-597.

Yoshikawa, T. (2000). Force control of robot manipulators. Proc. of the 2000 IEEE Int. Conf. Robotics \& Automation, San Francisco, CA (pp.220-226).

Zhang, F., Dawson, D.M., de Queiroz, M.S., \& Vedagarbha, P. (1997). Boundary control of the Timoshenko beam with freeend mass/inertial dynamics. Proc. of the 36th IEEE Conf. on Decision and Control, San Diego, CA (pp.245-250).

\section{Appendix A. Proof of estimate (33)}

We prove estimate (33). From the equation $u_{i j}\left(x_{i}\right)=\int_{0}^{x_{i}} u_{i j}^{\prime}(s) \mathrm{d} s+u_{i j}(0)$, the Cauchy-Schwartz inequality, and (36), we obtain

$\left\|u_{i j}\right\|_{L^{2}}^{2} \leq \gamma_{1}\left\{\left\|u_{i j}^{\prime}\right\|_{L^{2}}^{2}+\left|u_{i j}(0)\right|^{2}\right\}$

where $\gamma_{1}$ is a positive constant. On the other hand, integrating the equation $\left|u_{2 i}-u_{1 i}^{\prime}\right|^{2}=\left|u_{2 i}\right|^{2}-2 \operatorname{Re}\left(u_{2 i} u_{1 i}^{\prime}\right)+\left|u_{1 i}^{\prime}\right|^{2}$ and using the inequality $2|a||b| \leq \delta|a|^{2^{2}}+|b|^{2} / \delta$ for $a, b \in \mathrm{C}$, $\delta \in \mathrm{R}^{+}$, gives

$\left\|u_{2 i}-u_{1 i}^{\prime}\right\|_{L^{2}}^{2} \geq(1-\delta)\left\|u_{2 i}\right\|_{L^{2}}^{2}+(1-1 / \delta)\left\|u_{1 i}^{\prime}\right\|_{L^{2}}^{2}$.

Thus, we obtain the following using (A.1) and (A.2): for $\left(u_{1 i}, u_{2 i}\right) \in H^{1}(0, l) \times H^{1}(0, l)$

$$
\begin{aligned}
\left\|\left(u_{1 i}, u_{2 i}\right)\right\|_{1}^{2} \geq & \frac{E I_{i}}{2}\left\|u_{2 i}^{\prime}\right\|_{L^{2}}^{2}+\frac{K_{i}}{2}(1-\delta)\left\|u_{2 i}\right\|_{L^{2}}^{2} \\
& +\frac{K_{i}}{2}\left(1-\frac{1}{\delta}\right)\left\|u_{1 i}^{\prime}\right\|_{L^{2}}^{2}+\frac{k_{1 i}}{2}\left|u_{2 i}(0)\right|^{2}+\frac{k_{4}}{4}\left|u_{1 i}(0)\right|^{2} \\
\geq & \gamma_{2}\left\{\left\|u_{2 i}^{\prime}\right\|_{L^{2}}^{2}+\left|u_{2 i}(0)\right|^{2}\right\}+\frac{K_{i}}{2}(1-\delta)\left\|u_{2 i}\right\|_{L^{2}}^{2} \\
& +\gamma_{3}\left\{\left\|u_{1 i}^{\prime}\right\|_{L^{2}}^{2}+\left|u_{1 i}(0)\right|^{2}\right\}+\frac{E I_{i}}{4}\left\|u_{2 i}^{\prime}\right\|_{L^{2}}^{2} \\
& +\frac{K_{i}}{4}\left(1-\frac{1}{\delta}\right)\left\|u_{1 i}^{\prime}\right\|_{L^{2}}^{2} \\
\geq & \left\{\frac{\gamma_{2}}{\gamma_{1}}+\frac{K_{i}}{2}(1-\delta)\right\}\left\|u_{2 i}\right\|_{L^{2}}^{2}+\frac{\gamma_{3}}{\gamma_{1}}\left\|u_{1 i}\right\|_{L^{2}}^{2} \\
& +\frac{E I_{i}}{4}\left\|u_{2 i}^{\prime}\right\|_{L^{2}}^{2}+\frac{K_{i}}{4}\left(1-\frac{1}{\delta}\right)\left\|u_{1 i}^{\prime}\right\|_{L^{2}}^{2},
\end{aligned}
$$

where $\gamma_{2}=\min \left\{E I_{i} / 4, k_{1 i} / 2\right\}$, and $\gamma_{3}=\min \left\{K_{i}(1-1 / \delta) / 4, k_{4} / 4\right\}$. Here, if we set $1<\delta<2 \gamma_{2} /\left(K_{i} \gamma_{1}\right)+1$, we can obtain the positive constant $\gamma_{4}$ satisfying

$\left\|\left(u_{1 i}, u_{2 i}\right)\right\|_{1}^{2} \geq \gamma_{4}\left\|\left(u_{1 i}, u_{2 i}\right)\right\|_{2}^{2}$.

Here, (A.4) means the estimate (33). 